\title{
KEK digital accelerator
}

\author{
T. Iwashita, ${ }^{1}$ T. Adachi, ${ }^{1,2}$ K. Takayama,,${ }^{1,2,3}$ K. W. Leo, ${ }^{2}$ T. Arai, ${ }^{1}$ Y. Arakida,${ }^{1,2}$ M. Hashimoto, ${ }^{1}$ E. Kadokura, ${ }^{1}$ \\ M. Kawai, ${ }^{1}$ T. Kawakubo, ${ }^{1}$ Tomio Kubo, ${ }^{1}$ K. Koyama, ${ }^{1}$ H. Nakanishi, ${ }^{1}$ K. Okazaki, ${ }^{4}$ K. Okamura, ${ }^{1,2}$ H. Someya, ${ }^{1}$ \\ A. Takagi, ${ }^{1,2}$ A. Tokuchi, ${ }^{5}$ and M. Wake ${ }^{1,2}$ \\ ${ }^{1}$ High Energy Accelerator Research Organization (KEK), 1-1 Oho, Tsukuba, Ibaraki 305-0801, Japan \\ ${ }^{2}$ The Graduate University for Advanced Studies, Hayama, Miura, Kanagawa 240-0193, Japan \\ ${ }^{3}$ Tokyo Institute of Technology, Nagatsuda, Kanagawa 152-8550, Japan \\ ${ }^{4}$ Nippon Advanced Technology Co., Ltd., Tokaimura, Naka, Ibaraki 319-1112, Japan \\ ${ }^{5}$ Pulse Power Laboratory, Kusatsu, Shiga 520-0806, Japan \\ (Received 5 December 2010; published 20 July 2011)
}

The High Energy Accelerator Research Organization KEK digital accelerator (KEK-DA) is a renovation of the KEK $500 \mathrm{MeV}$ booster proton synchrotron, which was shut down in 2006. The existing $40 \mathrm{MeV}$ drift tube linac and rf cavities have been replaced by an electron cyclotron resonance (ECR) ion source embedded in a $200 \mathrm{kV}$ high-voltage terminal and induction acceleration cells, respectively. A DA is, in principle, capable of accelerating any species of ion in all possible charge states. The KEK-DA is characterized by specific accelerator components such as a permanent magnet X-band ECR ion source, a low-energy transport line, an electrostatic injection kicker, an extraction septum magnet operated in air, combined-function main magnets, and an induction acceleration system. The induction acceleration method, integrating modern pulse power technology and state-of-art digital control, is crucial for the rapid-cycle KEK-DA. The key issues of beam dynamics associated with low-energy injection of heavy ions are beam loss caused by electron capture and stripping as results of the interaction with residual gas molecules and the closed orbit distortion resulting from relatively high remanent fields in the bending magnets. Attractive applications of this accelerator in materials and biological sciences are discussed.

DOI: 10.1103/PhysRevSTAB.14.071301

PACS numbers: 29.20.dk, 41.75.Cn

\section{INTRODUCTION}

The High Energy Accelerator Research Organization KEK digital accelerator (DA) is a renovation of the former $500 \mathrm{MeV}$ rapid-cycle proton synchrotron (a booster for the KEK $12 \mathrm{GeV}$ PS). The KEK-DA is a small-scale induction synchrotron (IS) without a large injector. However, practical constraints such as the limited acceptance and spacecharge limited current in the downstream ring demand a certain injection velocity. Ions generated in an ion source embedded in a $200 \mathrm{kV}$ high-voltage terminal are injected into the KEK-DA.

The concept of an induction synchrotron was experimentally demonstrated in 2006 [1-3], using the KEK $12 \mathrm{GeV}$ PS. The properties of an induction synchrotron are contrasted with those of a conventional rf synchrotron in Fig. 1. Instead of an rf cavity, an induction cell (IC) is used as the acceleration device. This is only a one-to-one transformer, which is energized by the switching power supply generating pulse voltages. Two sorts of IC are utilized: one is only for acceleration and the other generates barrier voltages [4] for confinement of an ion bunch in the longitudinal

Published by the American Physical Society under the terms of the Creative Commons Attribution 3.0 License. Further distribution of this work must maintain attribution to the author(s) and the published article's title, journal citation, and DOI. direction. In other words, the IS is a functionally separated synchrotron in the longitudinal direction. A crucial feature of the IS is that voltage timing is controlled by an intelligent gate controller of solid-state switching elements, based on signals picked up at the bunch monitor. This operational performance allows acceleration of ions from extremely low velocity and is a reason why the DA does not require a large injector accelerator. Thus, the DA is capable of accelerating, in principle, any species of ion in any possible charge state. Details are given in Refs. [3,5].

Most of the device components of the KEK-DA shown in Fig. 2 are recycled from the KEK $500 \mathrm{MeV}$ booster, which was shut down in March 2006, after 35 years of operation as the injector of the KEK 12-GeV PS and as the driver of the neutron source for neutron diffraction experiments and cancer therapy and the proton driver for muon experiments. The main alterations are listed as follows: (i) construction of an electron cyclotron resonance (ECR) ion source and a $200 \mathrm{kV}$ high-voltage terminal; (ii) rearrangement of the low-energy beam transport (LEBT); (iii) introduction of an electrostatic chopper for injection pulse shaping and an electrostatic kicker for injection; (iv) replacement of the existing rf cavities by induction cells; (v) a change in the repetition frequency of the accelerator ring $(20 \mathrm{~Hz} \rightarrow 10 \mathrm{~Hz})$ to reduce wear on the acceleration device; and (vi) improvement in vacuum for longer beam lifetime. 


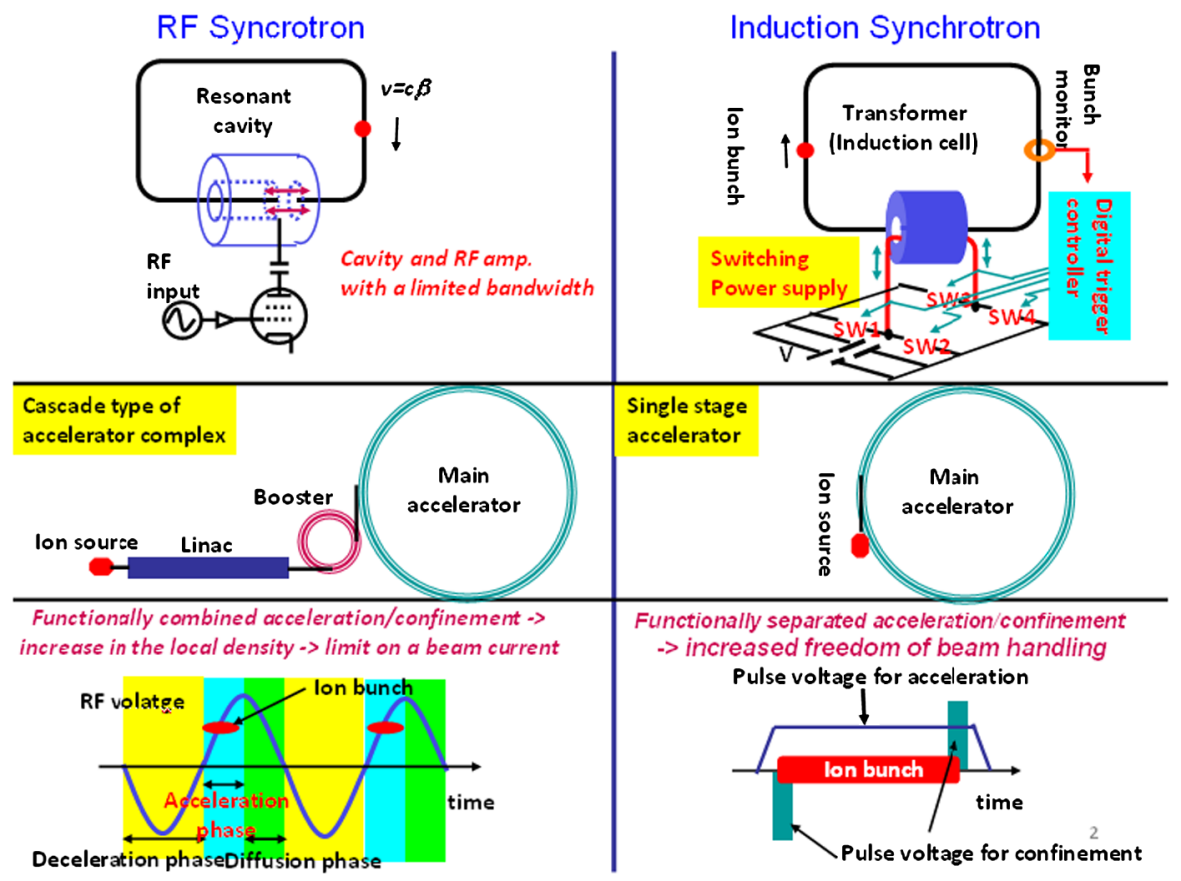

FIG. 1. Comparison between an rf synchrotron and an induction synchrotron (digital accelerator).

The term "digital accelerator" implies the following characteristics: (i) Since an induction cell is a simple one-to-one transformer driven by a switching power supply, there is no limitation of bandwidth on the lower side. (The maximum repetition rate is limited to megahertz because of the heat-deposit capacity of the solid-state switching element.) (ii) The induction cell is excited by a pulse current and generates a constant flat voltage, which is triggered every turn by a gate-control signal created by the digital signal processor (DSP) from a pickup signal in a fast bunch monitor. (iii) Acceleration voltage in a conventional rapid-cycle synchrotron must be varied sinusoidally. In the KEK-DA, acceleration is realized by controlling the density of the acceleration voltage pulse through an acceleration cycle.

In the following sections the KEK-DA subsystems are described. In Sec. II, we describe the electron cyclotron resonance ion source (ECRIS) and high-voltage terminal.

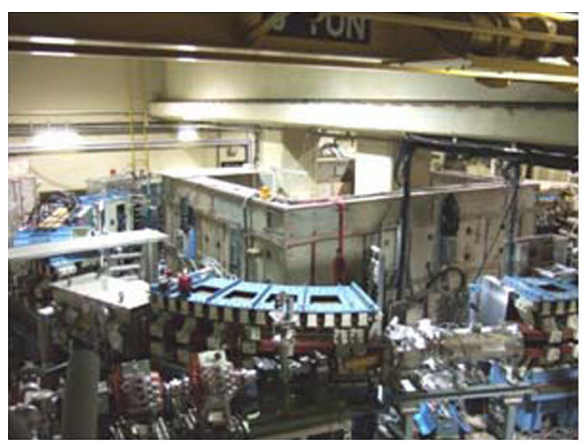

200 kV ECR Ion Source

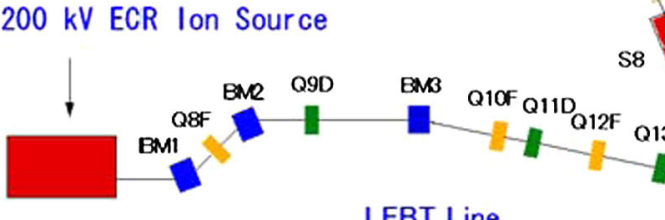

LEBT Line
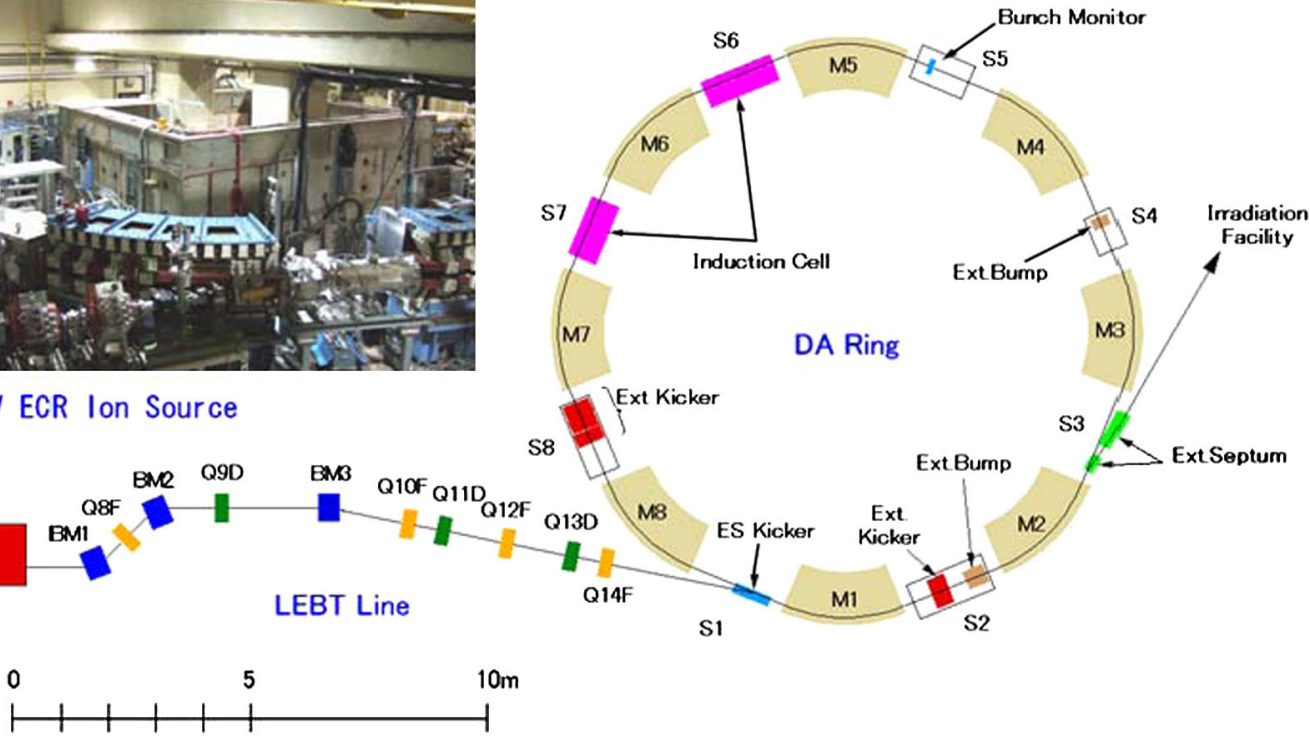

FIG. 2. Outline of the KEK digital accelerator and the recent photograph. 


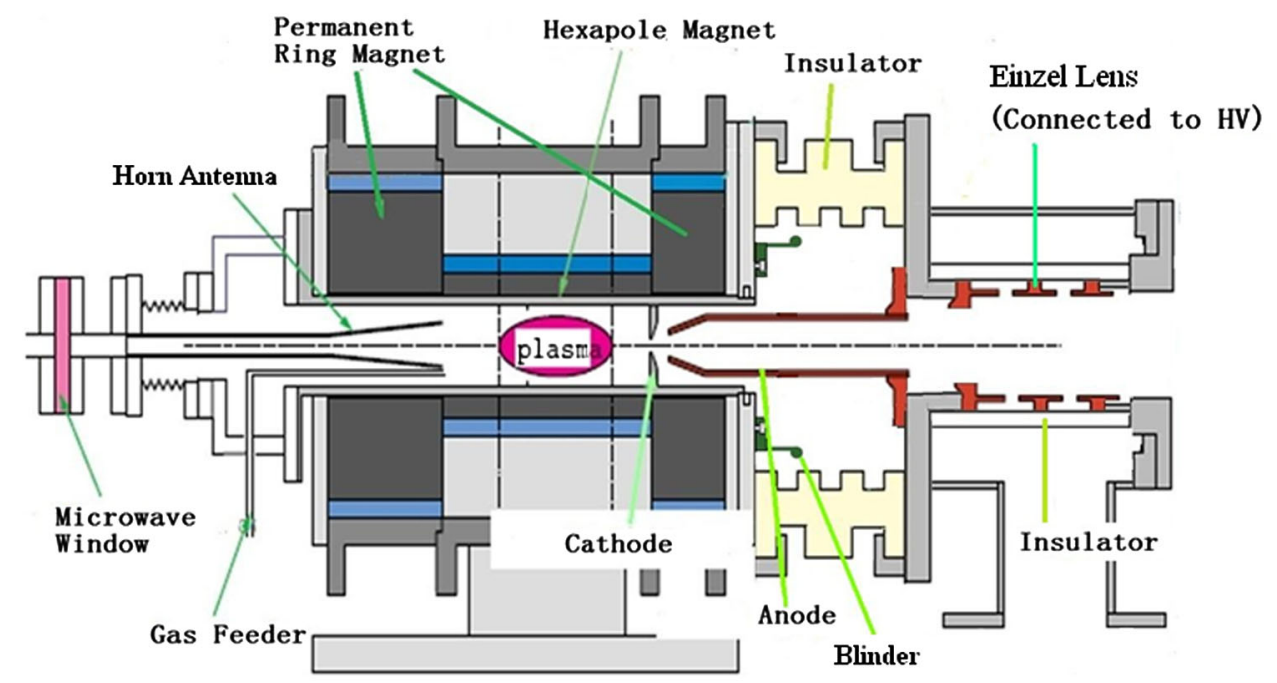

FIG. 3. Outline of ECRIS with the extraction system downstream.

Sections III, IV, V, and VI are dedicated to the LEBT, the DA ring, the injection and extraction systems, and the vacuum system, respectively. In Sec. VII, we explain beam acceleration in the DA ring in detail. There are intrinsic or severe issues such as space-charge limitations, COD caused by remanent fields, and interactions between circulating ions and residual gases, which are originated from the operation of the KEK-DA at an extremely low energy. At the expense of these handicaps, the KEK-DA makes it possible to provide various ion species without a large and expensive injector. In Sec. VIII, we discuss these issues related to beam dynamics and show how we can overcome such shortcomings. Planned beam parameters and applications are presented in Sec. IX. The last section summarizes this paper.

\section{ELECTRON CYCLOTRON RESONANCE ION SOURCE (ECRIS)}

\section{A. Outline of ECRIS}

Figure 3 shows the design of ECRIS. It consists of two permanent ring magnets, permanent hexapole magnets, the return yoke, the microwave horn antenna, and the extraction system. Microwaves of $9.35 \mathrm{GHz}$ are provided from a traveling wave tube (TWT) amplifier with a maximum power of $700 \mathrm{~W}$. As continuous wave operation had been envisaged, the plasma chamber was originally designed with water cooling channels. As a result, the aperture diameter of $4 \mathrm{~cm}$ was larger than that of similar X-band permanent ECRISs, such as Nanogan [6]. Since the KEKDA is operated at $10 \mathrm{~Hz}$, the injection of ion beams at the same repetition rate is expected. Ion pulses are generated in pulse mode, in which the TWT amplifier is gated by an external pulse generator to fire a 5-ms microwave pulse at $10 \mathrm{~Hz}$. In this mode of operation, water cooling is not required. Consequently, the large aperture plasma chamber was replaced by a small and thin aperture chamber of $3 \mathrm{~cm}$ in diameter. The space left between the magnet inner surface and outer surface of the plasma chamber is occupied by an additional thin hexapole magnet. A blinder helps to protect against metal ions spattering on the surface of the insulating ceramic pipe. The position of the horn antenna aperture is optimized for matching.

\section{B. Magnets}

It is well known that the high axial mirror ratio and a strong radial field inside the plasma chamber are
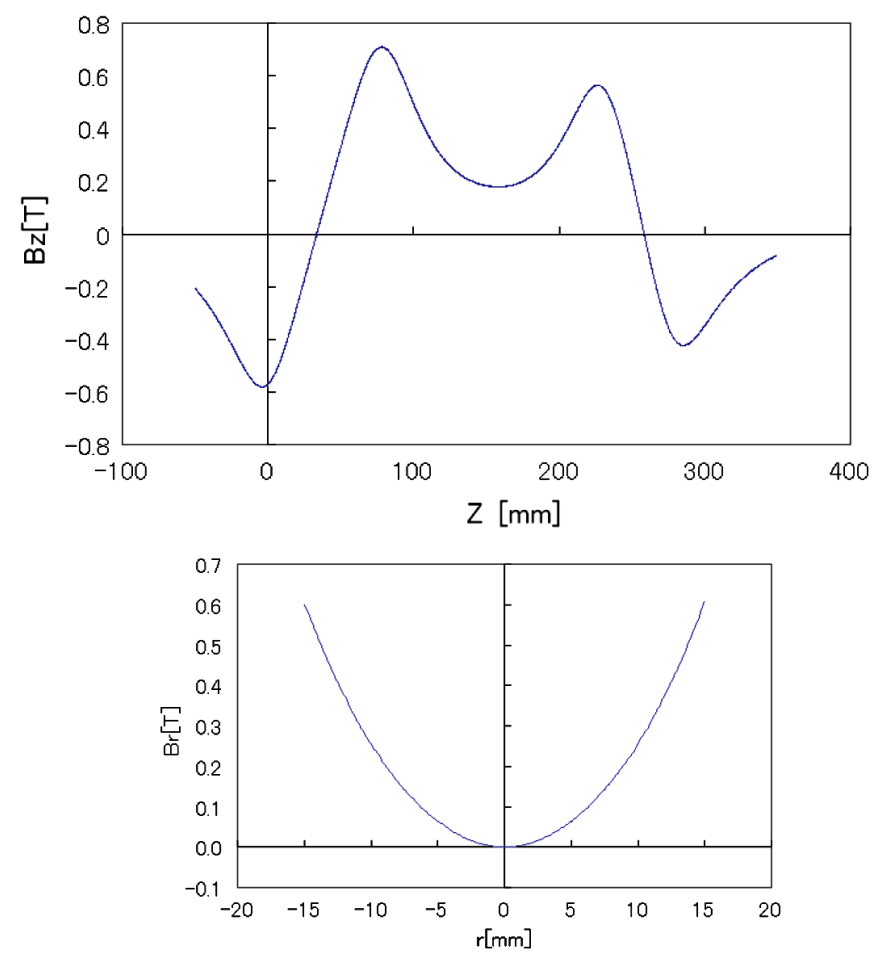

FIG. 4. Field distribution measured through the ECRIS, (top) $B_{z}(z)$ and (bottom) $B_{r}(r)$ at the minimum $B_{z}$. 

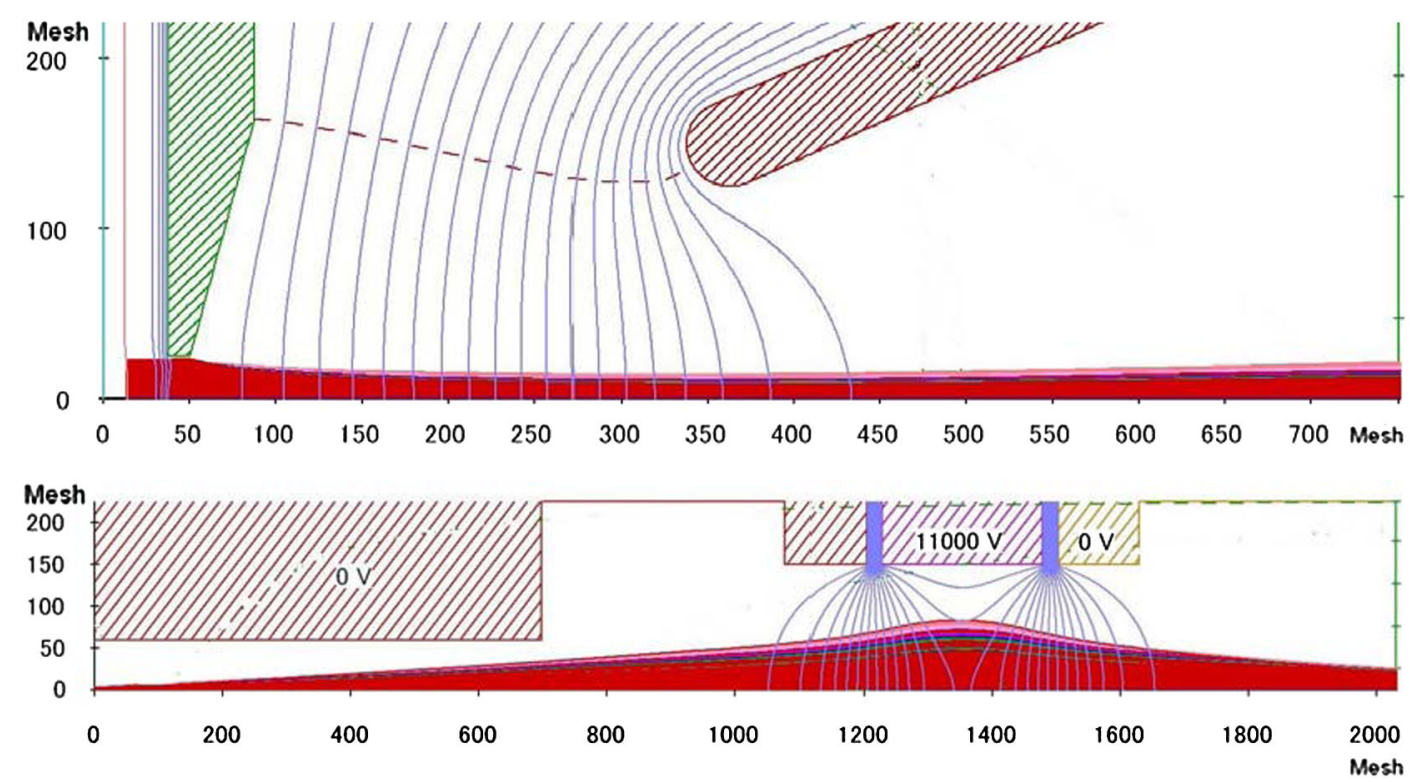

FIG. 5. Beam envelope from cathode to anode and from anode through Einzel lens (IGUN simulation result). Units are mesh size in simulation (20 $\mu \mathrm{m}$ in this calculation).

important [7]. For our current ECRIS, the first peak flux density $\left(B_{\text {peak } 1}\right)$ is $0.71 \mathrm{~T}$, the second one $\left(B_{\text {peak } 2}\right)$ is $0.565 \mathrm{~T}$ and the radial flux density $\left(B_{r}\right)$ on the inner surface of the plasma chamber $(r=15 \mathrm{~mm})$ is $0.6 \mathrm{~T}$. The resonance flux density $\left(B_{\mathrm{ECR}}\right)$ is $0.33 \mathrm{~T}$ for a frequency of $9.35 \mathrm{GHz}$. An empirical rule [7] tells us $B_{r} \sim 2 B_{\mathrm{ECR}}$ is desired. The actual field strength is a bit lower than this condition. The measured field distribution along the $z$, $B_{z}(z)$, and radial distribution $B_{r}(r)$ are plotted in Fig. 4 .

\section{Extraction system}

The IGUN [8] code has been used to simulate the beam orbits from the cathode hole to the anode and from the anode through the Einzel lens (see Fig. 5). The beam emittance and phase space plot are generated from the IGUN output. IGUN simulations based on the Langmuir-Child law [9] give a space-charge limited ion current if the relative fractional ratio of charge states is assumed. In our calculations, the experimentally obtained fractional ratios were used. Consequently, the estimated space-charge limited current was in good agreement with the experimentally obtained total ion current, which is monitored with a Faraday cup placed just after the Einzel lens.

The lattice function at the beginning of the test bench can be evaluated from the IGUN output. It is straightforward to calculate the beam envelope along the beam transport line by using this initial value and the lattice parameters, which are uniquely determined by the voltage of the electrostatic $Q$ lenses. It is interesting to compare the actual beam profile on the $X-Y$ plane with the predicted result. For this purpose, a beam profile measurement was carried out using an emission plate of alumina placed at the end point

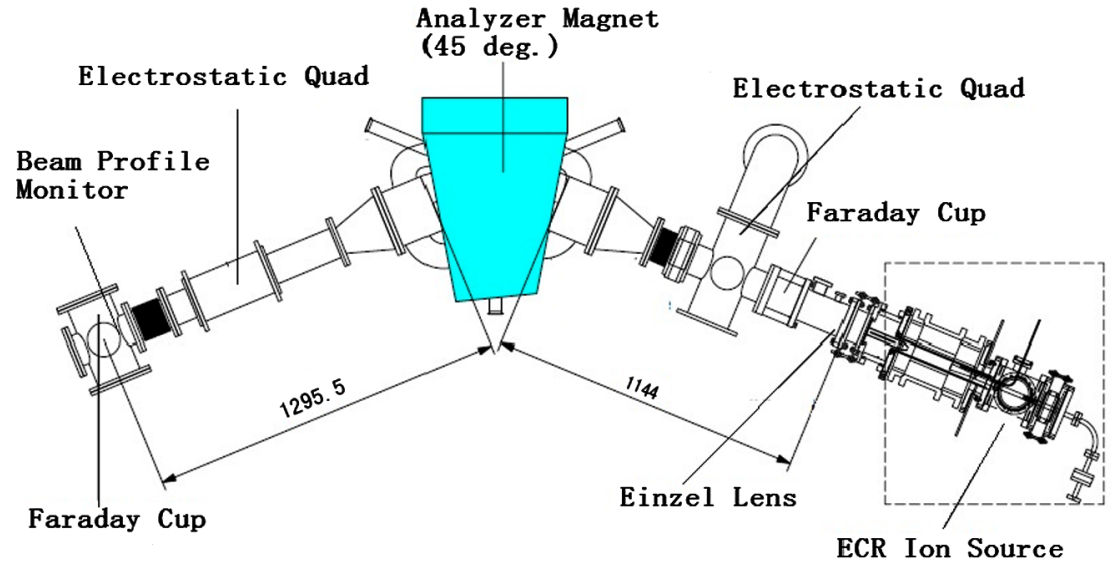

FIG. 6. Schematic overview of the test bench (unit is $\mathrm{mm}$ ). 
of the beam line. Basic qualitative features of the observed profile were consistent with the predicted ones.

\section{Einzel lens chopper}

The pulse width of the ion beam, which is injected into the KEK-DA, is uniquely determined from the revolution frequency in the ring: $5 \mu \mathrm{s}$ is typical for Ar ions, as will be discussed later. However, ion pulses delivered from the ECRIS are much longer-around 3-5 ms. This pulse length results from a fundamental buildup mechanism of highcharge-state ions under pulsed microwaves. This suggests that chopping of ion pulses delivered from the ECRIS is required. In general, chopping of a high energy beam is undesirable, because higher electric or magnetic fields are indispensable and secondary particles including $\mathrm{x}$ rays and electrons are generated from the surface of the vacuum chamber. To avoid these undesired features, an Einzel lens chopper has been developed at KEK. The high voltage of the middle electrode is rapidly altered using a negative pulse voltage with a rectangular shape in time, which is superimposed on the DC high voltage. This superimposing works like lowering a stopping barrier to allow ion flow downstream for a limited time. For achieve this, we have developed a solid-state Marx generator capable of generating a sharp rectangular pulse voltage. Details of the pulse driver, chopper simulation, and experiments are given elsewhere [10].

\section{E. Test bench results}

In order to investigate the operational performance of our ECRIS, including beam parameters and beam stability, the ECRIS test bench was used. It is divided into two portions: upstream and downstream. The upstream part consists of a diagnostic device (Faraday cup) placed just after the Einzel lens to measure the total ion current. The beam is focused by an electrostatic quadrupole doublet $(D)(F)$ and guided through the analyzer magnet to the downstream end. The downstream part consists of another doublet $(F)(D)$ and the diagnostic devices (Faraday cup and beam profile monitor) which are placed at the end point of the test bench, as shown in Fig. 6 .

The charge-state spectra of the extracted helium, carbon, nitrogen, oxygen, neon, and argon beams were obtained by monitoring the beam current in the downstream Faraday cup. Figure 7 shows the typical pulse shapes for individual charge states of argon at a gas flow rate of 0.05 SCCM (standard cubic centimeters per minute).

These results suggest that higher charge-state ions are created via their intermediate charge states within a time period of a few milliseconds. Absolute ion intensities have been obtained as shown in Table I.

\section{F. High-voltage terminal (HVT)}

The ECRIS is installed inside the high-voltage terminal (HVT). Based on the space-charge limit, the higher energy
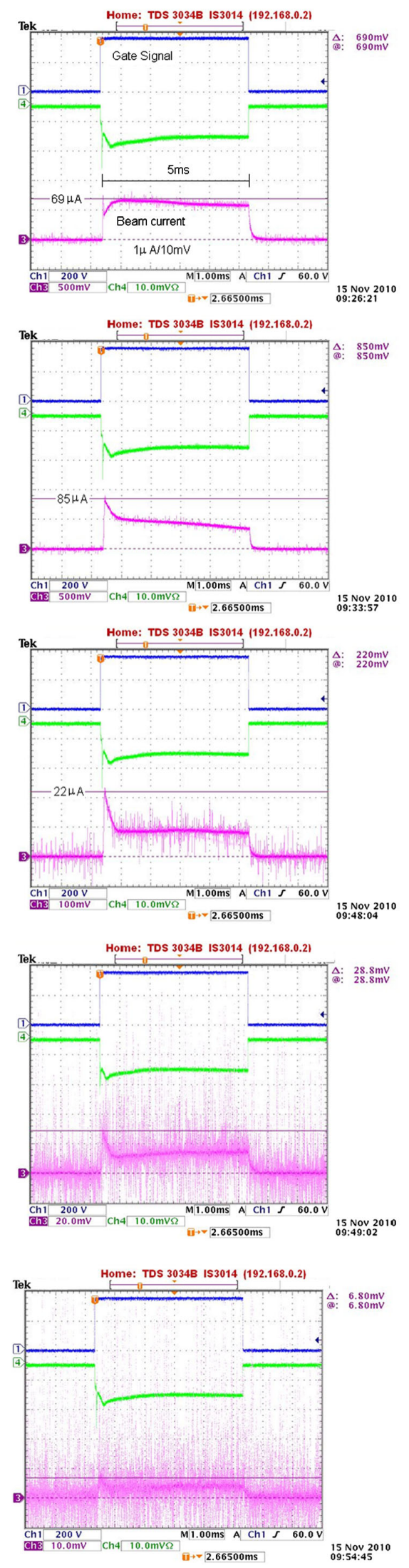

FIG. 7. Waveforms of the ion current from $Q=1$ to 5. Power/ pulse length: $600 \mathrm{~W} / 5 \mathrm{~ms}$. Upper trace is the reflection signal of microwaves for argon. 
TABLE I. Absolute ion intensities measured by Faraday cup (unit: $\mu \mathrm{A}$ ).

\begin{tabular}{rccccc}
\hline \hline$Q$ & $\mathrm{H}$ & $\mathrm{He}$ & $\mathrm{O}$ & $\mathrm{Ne}$ & $\mathrm{Ar}$ \\
\hline 1 & $>100$ & 300 & 118 & 142 & 69 \\
2 & & 4 & 72 & 54 & 85 \\
3 & & 18 & 4.8 & 22 \\
4 & & 1.25 & 0.24 & 3.5 \\
5 & & & $<0.1$ & 16 \\
6 & & 0.08 & $<0.1$ & 0.65 \\
7 & & & & 9 \\
8 & & 0.25 & & 3.2 \\
9 & & & $\sim 0.1$ & \\
10 & & & $\sim 0.1$ & 1.4 \\
\hline \hline
\end{tabular}

an ion reaches before injection, the higher intensity the beam can achieve (see Sec. X). An ion beam of chargestate $Q$ has a kinetic energy of $Q e V$, where $V$ is the sum of the extraction voltage and the terminal voltage. The HVT box, which is manufactured by a private company [11], has six insulating stands. A SPERMAN Cockloft-Walton highvoltage generator provides $200 \mathrm{kV}$. Low-voltage power for inner devices is supplied through an insulating transformer. The $200 \mathrm{kV}$ acceleration column is made of ceramics, and the $200 \mathrm{kV}$ is shared among three electrodes (see Fig. 8)
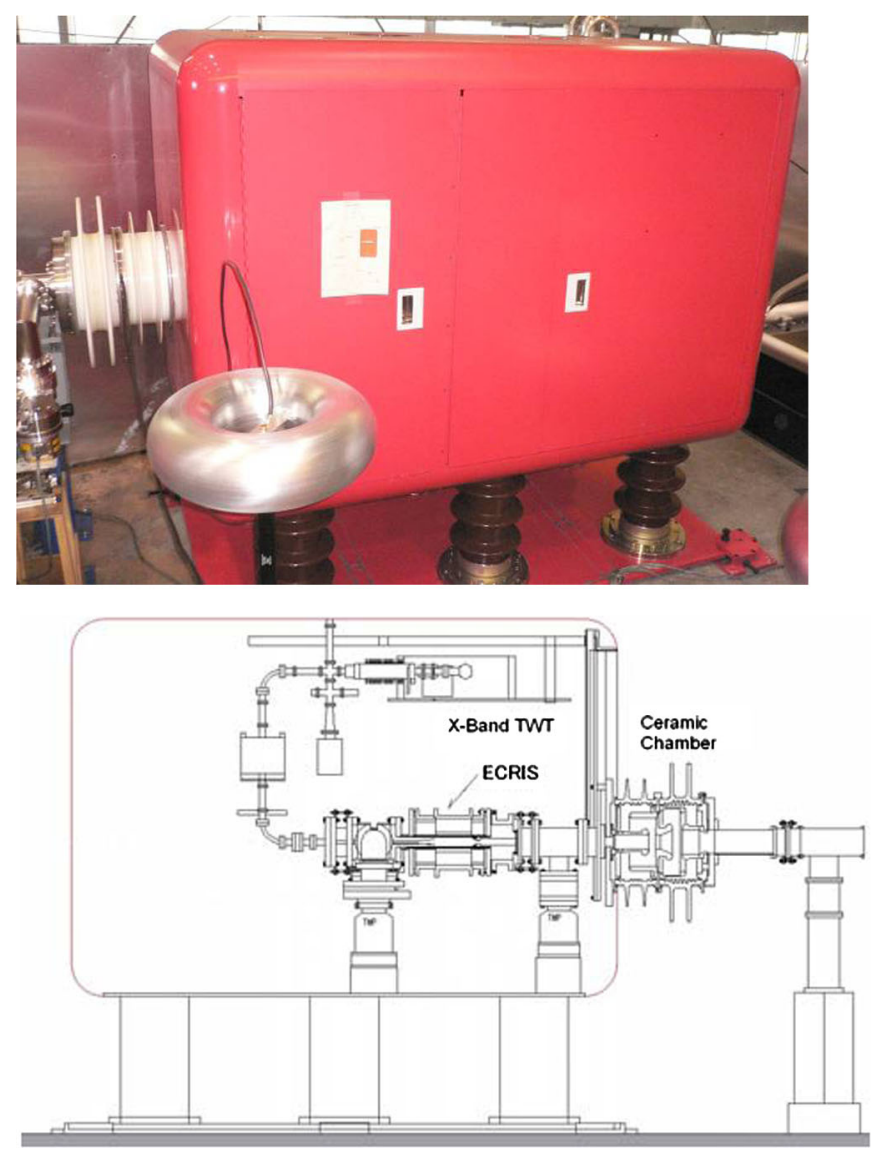

FIG. 8. A photograph of the HVT with an acceleration column and a diagram of its inner layout.

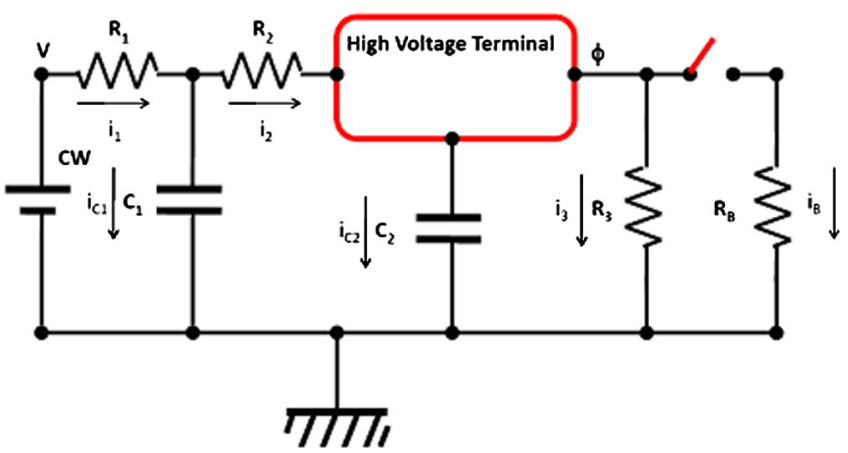

FIG. 9. Equivalent circuit of the HVT used to estimate voltage stability. $R_{1}$ and $R_{2}$ are the resistances of feeder line. $R_{3}$ is the resistance of the ceramic chamber. $R_{B}$ is the resistance seen by the beam current. $C_{1}$ is a capacitance inserted to stabilize the voltage. $C_{2}$ is the stray capacitance of the HVT.

estimated by using Femtet [12] simulation software. An expected voltage drop is given as a function of capacitance $C_{1}$. It turns out that in the case with $C_{1}$ beyond $30 \mathrm{nF}$ the voltage drop in the pulse length is less than $0.5 \%$, which is a tolerable level. Thus, the capacitance of $C_{1}=30 \mathrm{nF}$ has been connected between the HVT and the ground earth.

A drop in the acceleration voltage in the HVT is predicted to be associated with pulsed ion extraction. Assuming an ion beam current of $2.5 \mathrm{~mA}$, a pulse width of $5 \mathrm{~ms}$, and a period of $100 \mathrm{~ms}$, the change in the DC voltage level of the $200 \mathrm{kV}$ HVT can be estimated using the model shown in Fig. 9. Electrostatic parameters are obtained through actual measurements. A stray capacitance of HVT $\left(C_{2}\right)$ is estimated by using Femtet [12] simulation software. An expected, voltage drop is given as a function of capacitance $C_{1}$. It turns out that in the case with $C_{1}$ beyond $30 \mathrm{nF}$ the voltage drop in the pulse length is less than $0.5 \%$, which is a tolerable level. Thus, the capacitance of $C_{1}=30 \mathrm{nF}$ has been connected between the HVT and the ground earth.

\section{LOW-ENERGY BEAM TRANSPORT (LEBT)}

A low-energy beam transport (LEBT) is constructed downstream of the HVT. The LEBT is divided into two regions, which we call regions I and II (Fig. 10).

Region I has axial symmetry and consists of the Einzel lens located just after the extraction electrode, the $185 \mathrm{kV}$ acceleration column with the inner focusing electrodes and the beam drift space up to the entrance of the separation magnet. In this region, an Ar beam contains ions of all charge states $(+1$ to +8$)$. The space-charge limited ion current and beam envelope are calculated using the IGUN code, assuming the experimentally obtained fractions for $\mathrm{Ar}^{1+}$ to $\mathrm{Ar}^{8+}$.

Region II is from the entrance of the separation magnet to the end of the electrostatic injection kicker. It has an asymmetric focusing feature. The lattice has been designed with the edge focusing in the two dipole magnets taken into 


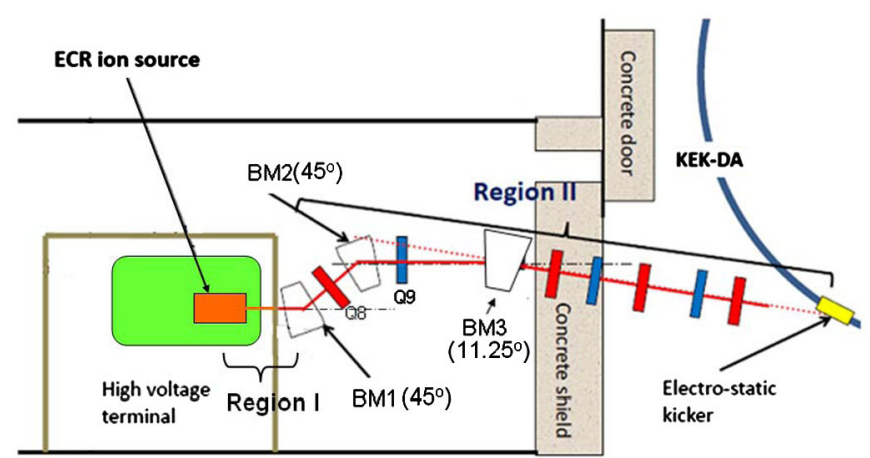

FIG. 10. LEBT line from the ECRIS to the DA ring.

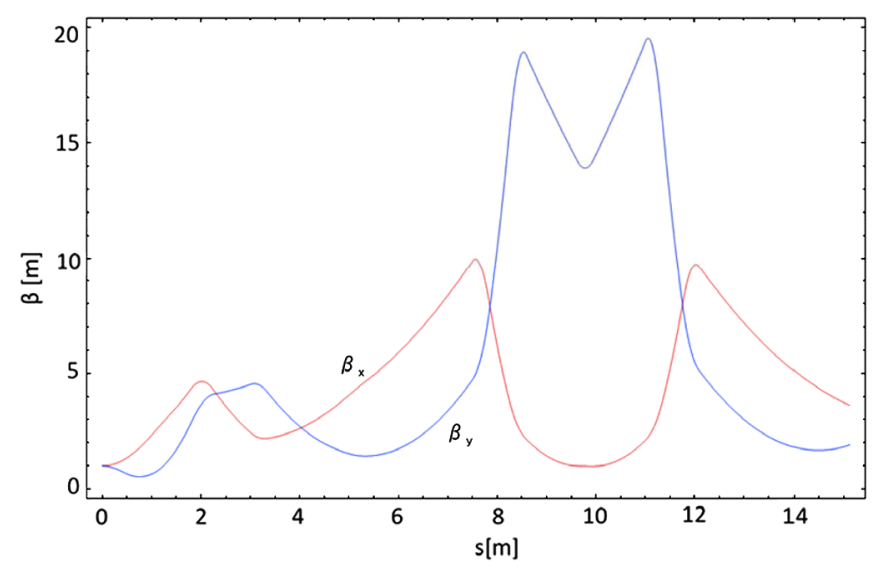

FIG. 11. Beta functions in the $x$ and $y$ directions.

account, so that the lattice function matches that of KEK-DA at the exit of the injection kicker. The beta functions in the $x$ and $y$ directions are shown in Fig. 11.

\section{DIGITAL ACCELERATOR RING}

\section{A. Lattice}

The DA ring consists of eight FDF type combinedfunction magnets. The lattice parameters are summarized in Table II. The beta functions and dispersion function are shown in Fig. 12.

\section{B. Main magnets}

Figure 13 shows a schematic view of the main magnets. The main magnets have an $F D F$ structure, in which a $D$ sector is sandwiched between $F$ sectors. The $D$ sector and the $F$ sectors are smoothly connected by a $T$ sector, which has a transient structure. All sectors are fabricated using an oriented low-carbon, low-silicon steel of $0.35 \mathrm{~mm}$ in thickness. The magnet parameters are listed in Table III.

\section{Power supply [13]}

There are eight magnets in the DA ring and one monitor magnet placed in the power supply building. All of them

TABLE II. DA lattice parameters.

Lattice (1 period)

Super period

Average radius

Bending radius

Magnet length

Length of the drift space

$Q_{X}$ (injection-extension)

$Q_{Y}$ (injection-extension)

$\beta_{X}(\max / \min )$

$\beta_{Y}(\max / \min )$

Chromaticity $(X / Y)$

Momentum compaction

Transition gamma

$\eta_{\max }$

are excited at a repetition rate of $10 \mathrm{~Hz}$ in a resonant network circuit, as shown in Fig. 14. The resonant network comprises three meshes, each of which contains a series inductance of three magnets and a parallel circuit consisting of a resonant capacitor and an inductance from a secondary winding of a choke transformer. The primary winding and all secondary windings of the choke have a common iron core, so that close coupling of each resonant

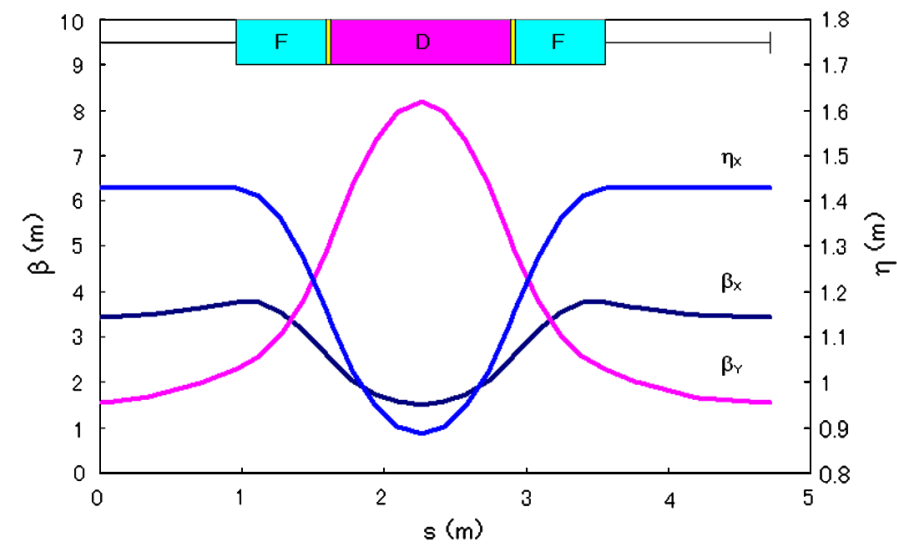

FIG. 12. Beta functions and dispersion function.
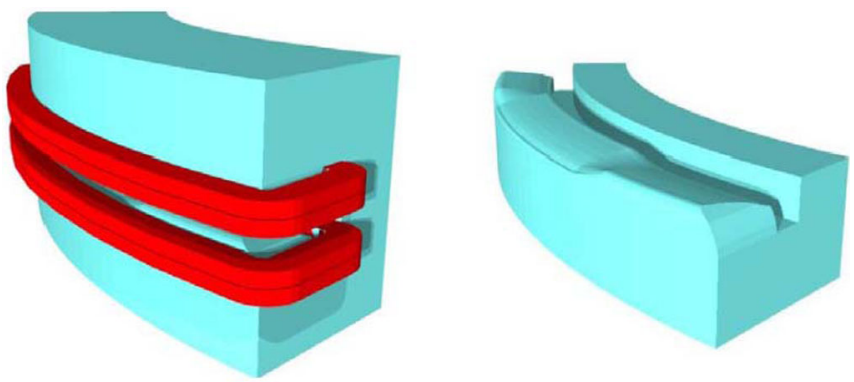

FIG. 13. Schematic view of the main magnet. In the right-hand figure, the upper half of the magnet core is omitted to show the pole shape. 
TABLE III. Parameters of main magnets.

Thickness of steel sheets

$0.35[\mathrm{~mm}]$

Gap height at pole center

Pole width

Number of turns (per pole)

$B_{\max }$

$6[\mathrm{~mm}]$

$300[\mathrm{~mm}]$

$$
20
$$

$1.1[\mathrm{~T}]$

Quadruple component $B^{\prime} / B(F / D)$

Sextuple component $B^{\prime \prime} / B(F / D)$

Length of $F$ sector

Length of $T$ sector

Length of $D$ sector

$3.664 /-3.664[1 / \mathrm{m}]$

$-0.615 /-0.934\left[1 / \mathrm{m}^{2}\right]$

$0.6313[\mathrm{~m}]$

$0.0352[\mathrm{~m}]$

$1.2588[\mathrm{~m}]$

mesh is realized. A DC power supply (DCPS) is connected to the choke transformer secondary windings in one mesh and feeds a bias current to all magnets. An AC power supply is connected to the choke's primary winding and provides AC power which is dissipated in the resonant circuit.
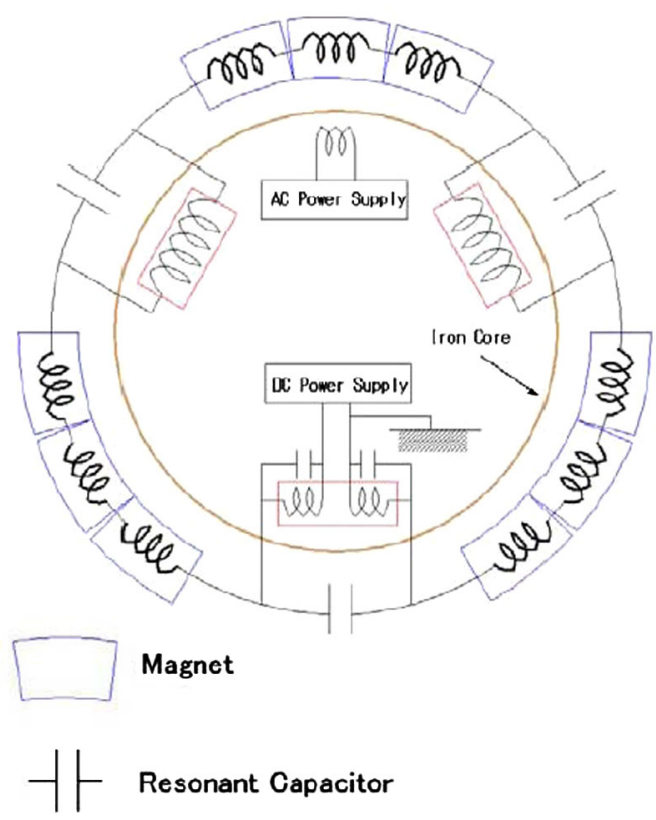

Secondary Winding of the Choke Transformer

FIG. 14. Resonant network for excitation of KEK-DA main magnets.
Since the resonant network is equivalent to the parallel resonant circuit shown in Fig. 15, the resonant frequency is given by

$$
\omega=\sqrt{\frac{L_{m}+L_{\mathrm{ch}}}{L_{m} L_{\mathrm{ch}} C}} .
$$

Here, $L_{m}$ is the series inductance of three magnets, $L_{\mathrm{ch}}$ the effective inductance of the choke secondary winding, and $C$ the capacitance of the resonant capacitor. $L_{\mathrm{ch}}$ is given by

$$
L_{\mathrm{ch}}=[1+(n-1) \kappa] L,
$$

where $L, \kappa$, and $n$ are the self-inductance of the choke secondary winding, coupling coefficient of mutual inductance between meshes and mesh number, respectively. In our case, $\kappa>0.995$ and $n=3$.

The magnetic field is a superposition of DC and AC fields: $B(t)=B_{\mathrm{DC}}-B_{\mathrm{AC}} \cos \omega t$. The original resonant network was tuned to resonate at $10 \mathrm{~Hz}$. In the current KEK-DA project, the acceleration voltage is limited by the number of available acceleration devices. To lower the required maximum voltage, the resonant frequency has been altered by rearranging the existing capacitor units. The resultant resonant frequency is measured to be $9.97 \mathrm{~Hz}$

\section{INJECTION AND EXTRACTION SYSTEM [14,15]}

\section{A. Electrostatic kicker}

\section{Design}

An injected argon beam of $5 \mu \mathrm{s}$ is deflected by the electrostatic kicker to merge into the DA ring orbit in straight section 1 (S1). The electrostatic kicker is a device that deflects the beam horizontally (see Fig. 16). Three subsidiary electrodes are inserted symmetrically between the anode (high-voltage electrode) and ground electrode. These electrodes are connected to each other by voltage dividing resistors to obtain a linear voltage drop, which ensures field homogeneity throughout beam passage.

\section{Power supply}

The kicker is excited by the pulse forming network shown in Fig. 17. It is necessary to turn off the voltage pulse quickly (within a single revolution) just after the injection to prevent the remaining field from disturbing

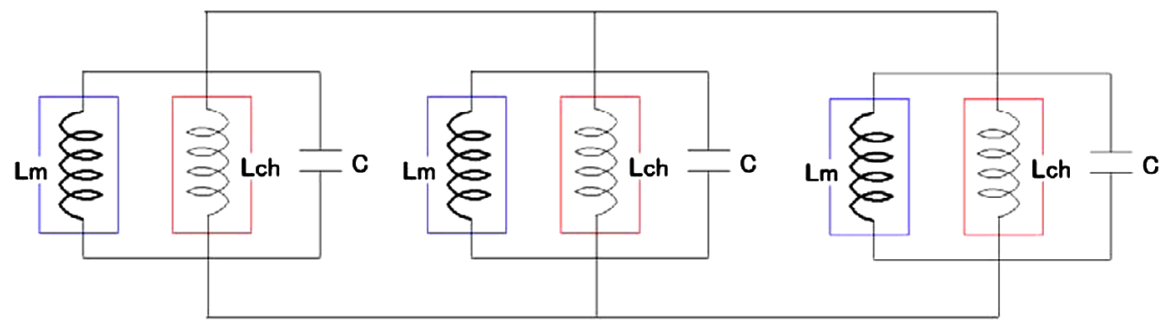

FIG. 15. Equivalent circuit for the resonant network. 

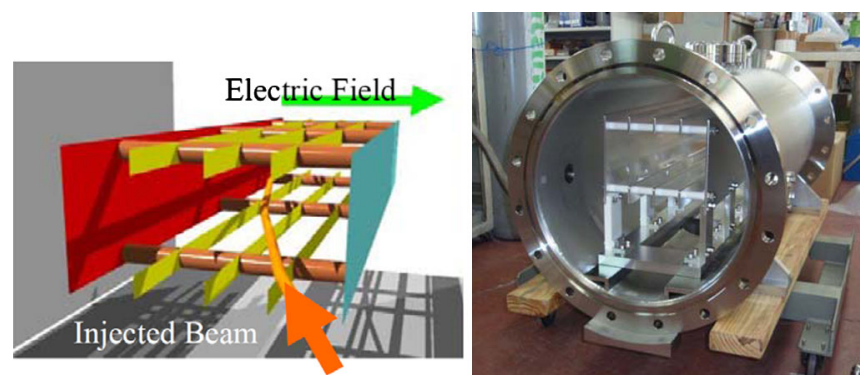

FIG. 16. Schematic view of the injection kicker (left) and a photograph of the kicker (right).

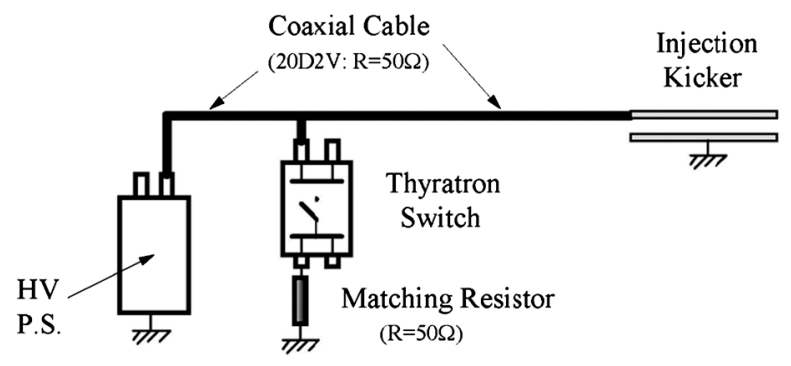

FIG. 17. Pulse forming network for the injection kicker.

the circulating beam. For this purpose, a matching resistor $(50 \Omega)$ to absorb reflection voltage is inserted. Table IV summarizes the specifications of the injection kicker.

\section{Simulation of the kicker field}

As stated in Sec. VB, it is important to maintain the homogeneity of the electrostatic field inside the kicker to avoid beam blowup. The field distribution was evaluated by TOSCA/OPERA3D, which is a simulation code for solving electrostatic problems.

In Fig. 18, the magnitudes of the calculated horizontal electric fields $E_{x}$ at the center of the kicker $(y=z=0)$ are plotted for three different situations as a function of the horizontal position $x$, where the origin $(x=0)$ corresponds to beam center and the anode voltage is fixed at $20 \mathrm{kV}$. It turns out that, to ensure field homogeneity, it is important for the subsidiary electrodes to share the voltage equally.

\section{Injection orbit simulation}

The phase space distribution of the beam has also been simulated using the calculated 3D field distribution of the injection kicker for case 1. The left-hand figure of Fig. 19

TABLE IV. Specifications of the injection kicker.

\begin{tabular}{lc}
\hline \hline Deflection angle & $11.25[$ degrees] \\
\hline Length & $800[\mathrm{~mm}]$ \\
Maximum voltage & $20[\mathrm{kV}]$ \\
Aperture $(\mathrm{W} \times \mathrm{H})$ & $200 \times 100\left[\mathrm{~mm}^{2}\right]$ \\
\hline \hline
\end{tabular}

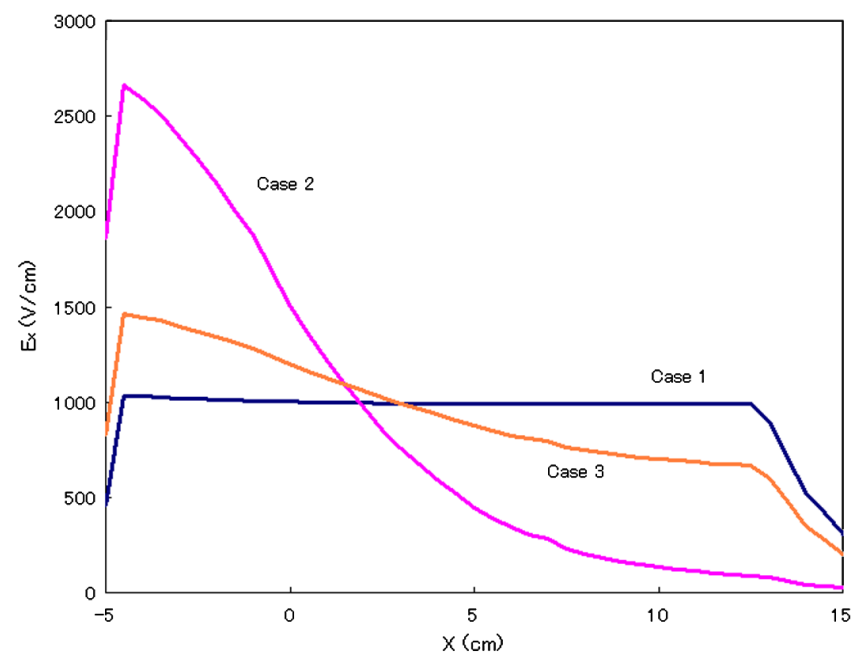

FIG. 18. Horizontal component of the electric field $E_{x}$ at the center of the kicker electrode $(y=z=0)$ vs $x$. In case 1 the subsidiary electrodes share the voltage equally. In case 2 each subsidiary electrode is floated. In case 3 all subsidiary electrodes are removed.

shows snapshots in the phase space, where the phase plot moves from the lower right to the upper left along the beam passage. The origin corresponds to the end of the injection kicker. Two right-hand figures show the beam distributions in the vertical phase space: the upper figure is for the beam at the entrance and the lower is at the exit of the kicker. The central orbit in the DA ring is $x=0.0$ and $y=0.0$ in each figure. These simulation results indicate that emittance blowup is negligibly small if the electric field of case 1 is realized.

\section{Voltage measurements}

To confirm the uniformity of the voltage distribution over time, the voltage was measured on the assembled injection kicker. The kicker was excited by a sinusoidal voltage source at operating frequencies of DC, $1 \mathrm{kHz}$, $10 \mathrm{kHz}, 100 \mathrm{kHz}$, and $1 \mathrm{MHz}$. The potential on an individual electrode was fixed by using dividing resistors of $1 \mathrm{M} \Omega$, which were connected to the outside of the vacuum chamber to avoid outgas from the extra devices. The voltage distribution shown in Fig. 20 exhibits nonlinearity beyond $10 \mathrm{kHz}$ and approaches a saturated distribution as the operating frequency increases.

The results indicate that the voltage distribution among electrodes strongly depends on the frequency of the exciting AC voltage. This implies that the existence of stray capacitances between electrodes and the ground strongly affects the voltage distribution as well as the dividing resistors. We estimated the stray capacitance which reproduces the frequency dependence of the measured voltage distribution by SPICE simulations. The estimated capacitances are around $50 \mathrm{pF}$ with respect to the ground or between subsidiary electrodes. 

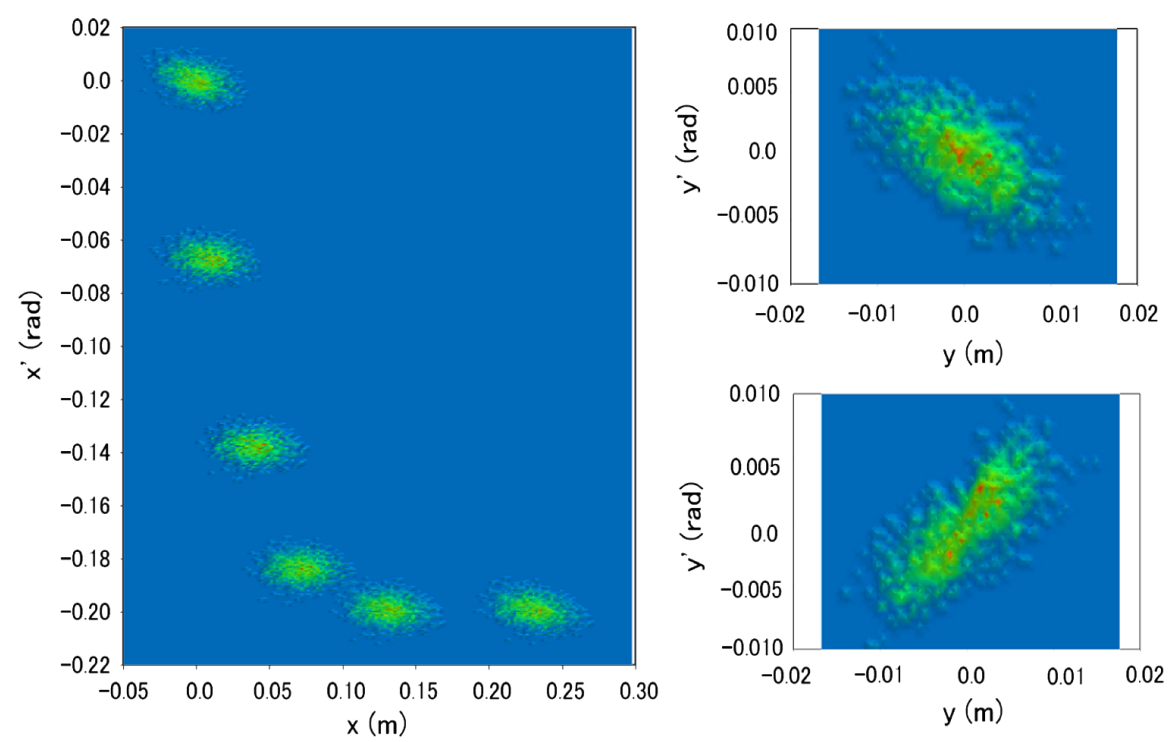

FIG. 19. Phase space distribution in the kicker field. In the left figure, the phase plot moves from the lower right to the upper left along the beam passage. The origin corresponds to the end of the injection kicker. Two right-hand figures show the beam distributions in the vertical phase space: the upper figure is for the beam at the entrance and the lower is at the exit of the kicker. The central orbit in the DA ring is $x=0.0$ and $y=0.0$ in each figure.

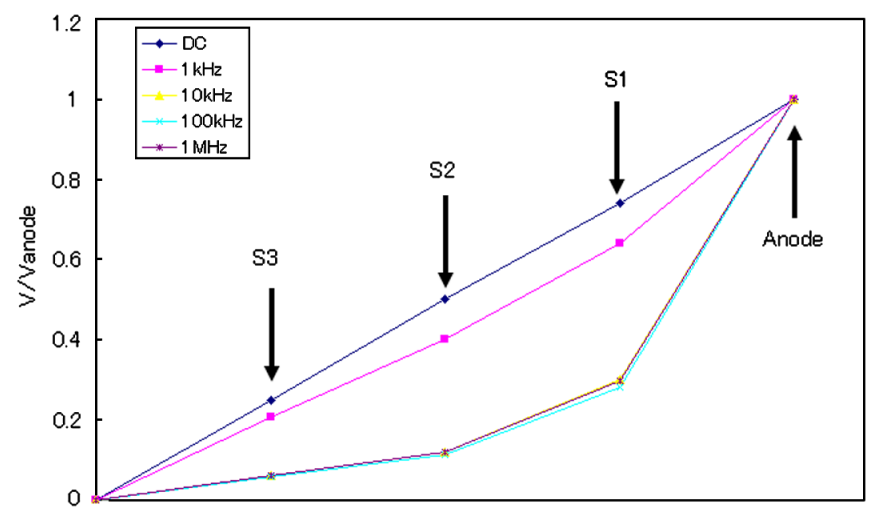

FIG. 20. Voltages measured at the anode and subsidiary electrodes S1, S2, and S3 (normalized by the anode voltage).

In order to ensure a linear voltage distribution even in the pulse operation of the kicker, a capacitance of $330 \mathrm{pF}$, which is sufficiently larger than the stray capacitance, was put in parallel with each dividing resistor. The measured output voltage is shown in Fig. 21. A uniform voltage distribution has been achieved even during pulse operation.

\section{B. Extraction system}

After the beam has been accelerated up to its maximum energy, it is extracted from the DA ring. The extraction system comprises two bump magnets, four kicker magnets, and two septum magnets. Figure 22 shows the extraction system and beam envelope in the corresponding region. The specifications of the bump magnets and kicker magnets, which had been used as the extraction system of the KEK $500 \mathrm{MeV}$ booster synchrotron, are available in the literature [13]. First, the bump magnets are excited just before the extraction to shift the circulating beam orbit nearer to the septum coil. Then, the excited kicker magnets kick the beam into the core gap of the septum magnets. Thus, a beam extracted from the DA ring is guided through the septum magnets to the transport line.

One of the important upgrades in KEK-DA was the modification of the septum magnets, which had been excited inside the vacuum chamber. Now they have been removed from the vacuum chamber and are excited in air [15]. This reduces outgas from magnet cores and excitation coils and reduces the total outgassing area. New vacuum ducts have been inserted into the pole gaps. Details of beam loss and vacuum are discussed in Sec. VIIIC. Figure 23 shows the septum magnet and the newly developed two vacuum ducts seen from upstream. The left duct is for the circulating beam and the right for the extracted beam. Both ducts are welded to a single flange upstream, while they are separated downstream so that an eddy current loop is not formed. The right duct is cooled by water to remove heat generated by eddy loss. The magnetic field in the duct was measured, and it was found that the contribution from eddy currents was sufficiently small.

The magnetic fields of the septum magnets were measured using a Hall element. Figure 24 shows the waveforms of the field measured at the center of the beam orbit inside the septum with and without the vacuum chamber. Although duct insertion causes a slight phase delay of the waveform, peak values are not affected. Figure 25 shows the peak field along the $z$ direction (beam axis) where $z=0 \mathrm{~cm}$ is defined as the entrance of the septum. In the region $z>5 \mathrm{~cm}$, the magnetic flux density is constant at $6.8 \mathrm{kG}$ and is independent of the presence of the duct. 


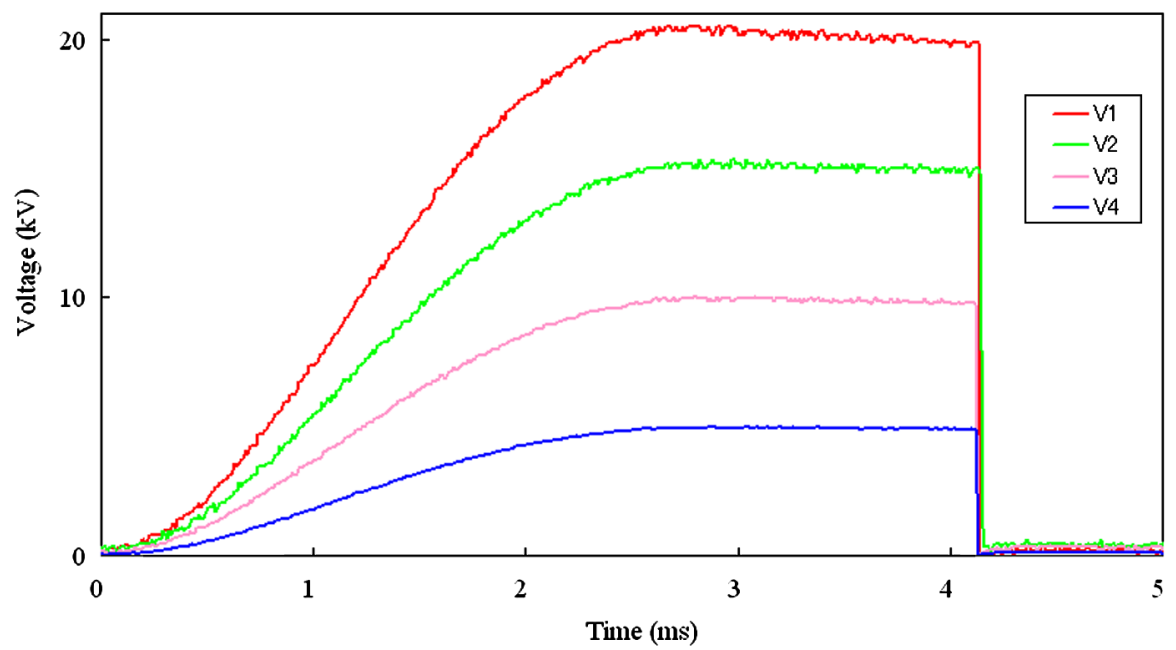

FIG. 21. Voltage waveforms measured at kicker electrodes. Voltages V1-V4 correspond to the voltages at the anode and the subsidiary electrodes S1-S3, respectively.

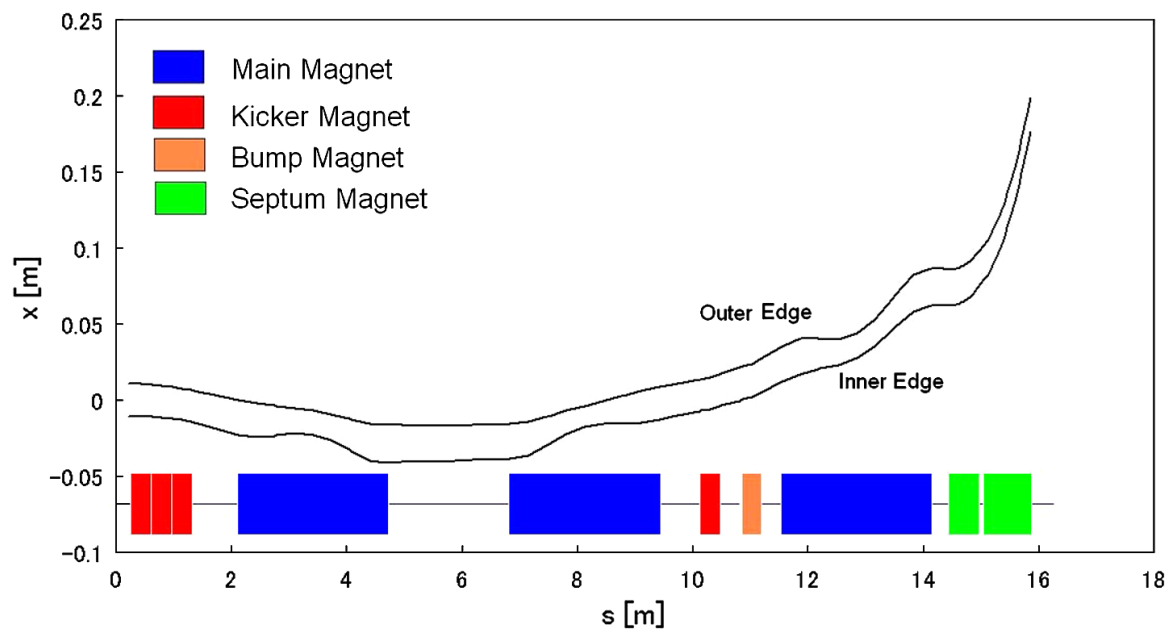

FIG. 22. Extraction system and the beam envelope.

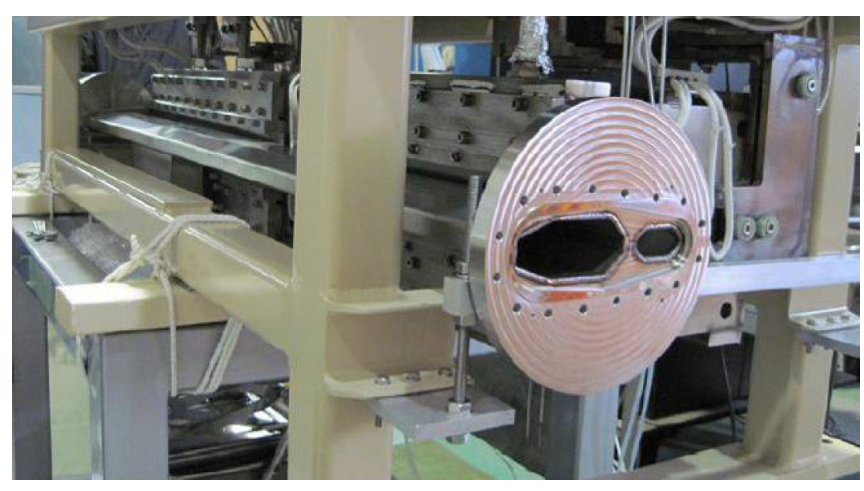

FIG. 23. Septum magnet and new vacuum ducts. The left duct is for the circulating beam and the right duct is for the extracted beam.

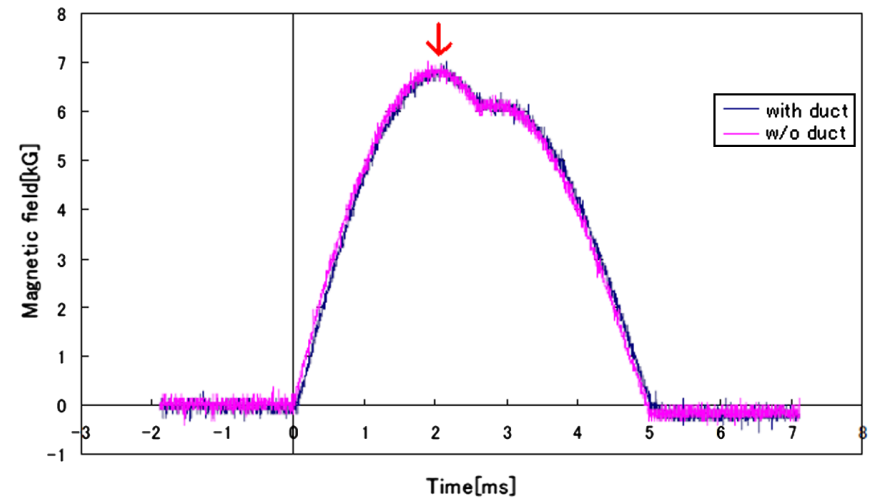

FIG. 24. Time profile of the septum magnetic fields. The arrow indicates the extraction timing. 


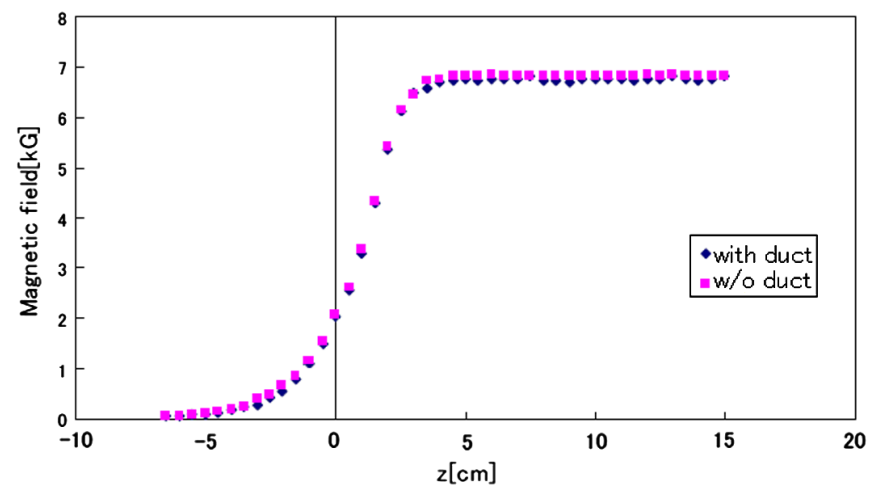

FIG. 25. Peak fields of the septum magnet along the beam axis.

\section{VACUUM SYSTEM}

An overview of the vacuum system is shown in Fig. 26. Pumps are installed in straight sections of the DA ring. There is a rotary pump $950 \mathrm{l} / \mathrm{m}$ ) for rough pumping, two

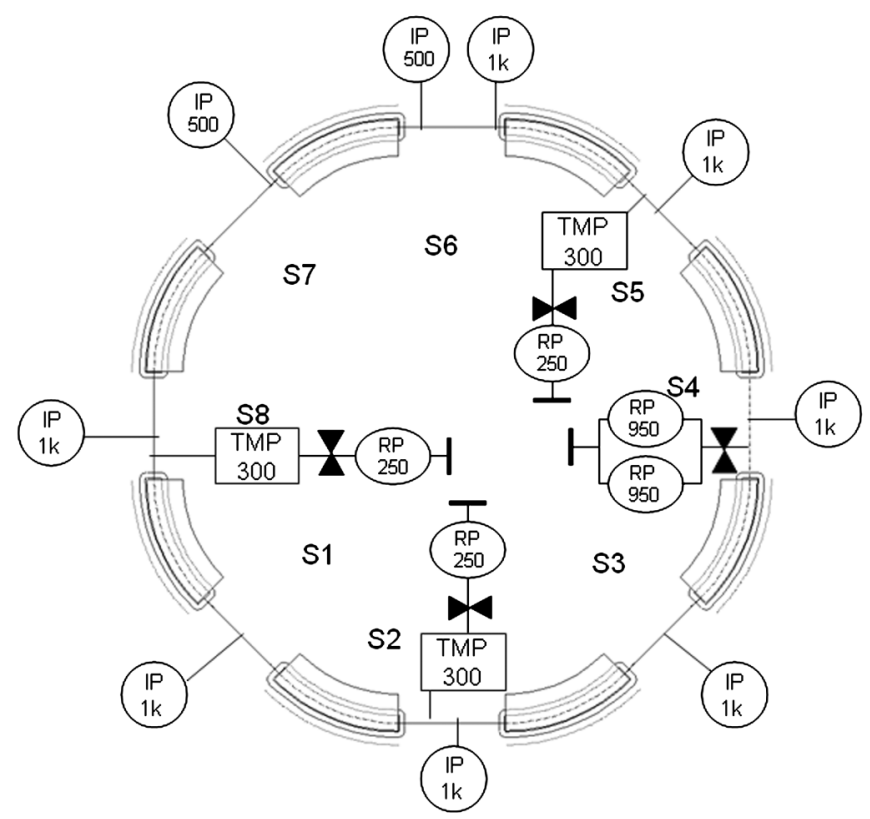

FIG. 26. Overview of the DA ring vacuum system. RP, TMP, and IP denote a rotary pump, turbo molecular pump, and ion getter pump, respectively. The numbers after the pump type are their pumping speed $(1 / \mathrm{min})$. turbo molecular pumps $(300 \mathrm{l} / \mathrm{m})$ for fine pumping. Under $10^{-4} \mathrm{~Pa}, 1000$ or $500 \mathrm{l} / \mathrm{s}$ ion pumps are used.

\section{BEAM ACCELERATION IN THE DA RING}

\section{A. Acceleration system}

An equivalent circuit for the induction acceleration system, which consists of a switching power supply and an acceleration cell, is shown in Fig. 27. A high-voltage pulse generated by the switching power supply (SPS) energizes the induction cell to accelerate the beam. The SPS and the induction cell are connected by a long coaxial cable to protect semiconductor switching elements inside the SPS from the high radiation dose in the accelerator tunnel. Details of each component are described below.

\section{Switching power supply (SPS) [16]}

The SPS, which modulates a DC voltage provided by the DC power supply, is operated in a full bridge circuit. The single switching arm seen in Fig. 28 actually consists of seven power MOSFETs (metal oxide semiconductor field effect transistor DE475-102N21A provided by IXIS rf Co. Ltd.) connected in series, which enables high-frequency switching at $1 \mathrm{MHz}$ with an output voltage of $2.5 \mathrm{kV}$ and peak current of $20 \mathrm{~A}$. The seven MOSFETs sharing the high voltage must be individually isolated from ground. The gates of these MOSFETs are turned on and off by gate signals, which are generated in the control system discussed later and delivered through optical fibers. The power for the MOSFET driving circuit is provided through a DC-DC converter with an extremely low capacitance, because the source-drain voltage balance between individual MOSFETs is strongly dependent on the effective capacitance in the circuit [17].

\section{Induction cell (IC) [18]}

We have developed two types of IC. Type A is a 1:1 transformer, in which a toroidal magnetic core is embedded. Its primary loop is connected to the SPS and the secondary loop is the beam orbit itself. The induced voltage along the secondary loop works as an acceleration voltage. As a matter of fact, the beam orbit is surrounded by a metallic vacuum chamber. The vacuum chamber

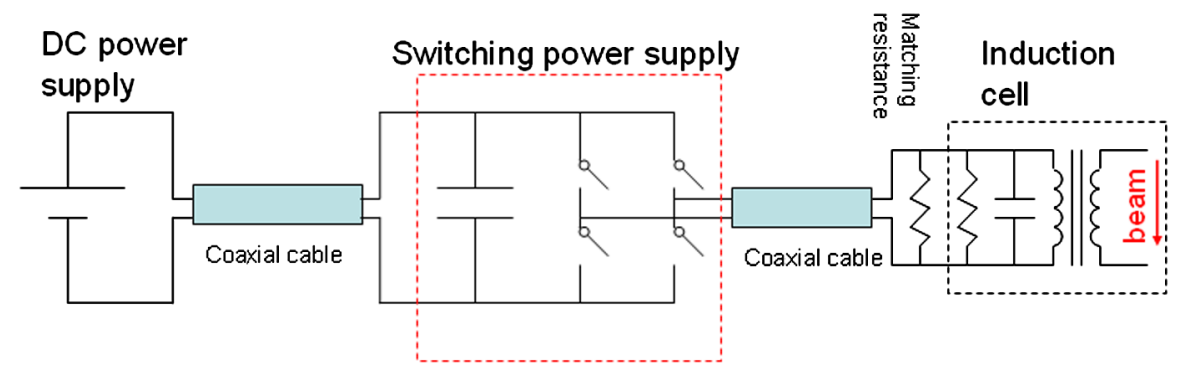

FIG. 27. An equivalent circuit for the induction acceleration system. 


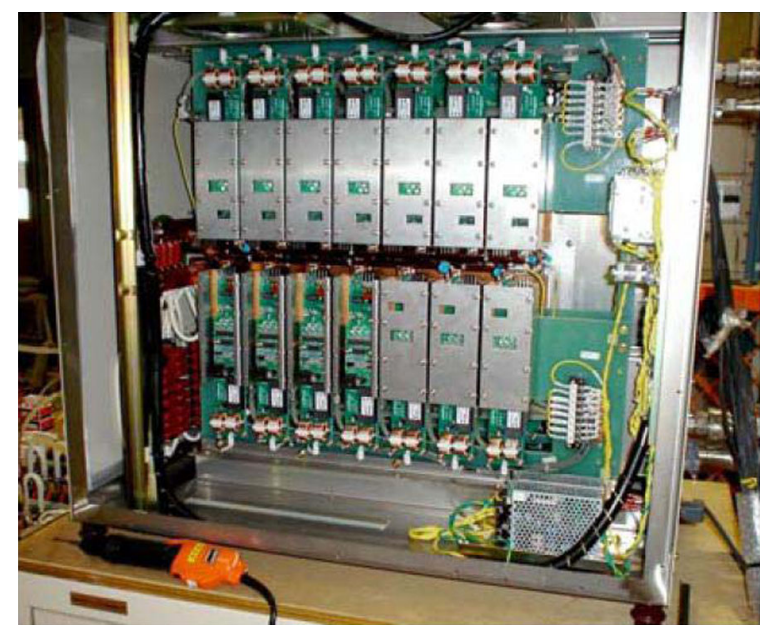

FIG. 28. A photograph of the SPS. There are seven MOSFETs in each arm.

inside the transformer has a ceramic gap which is welded to both edges of the vacuum chamber. Thus, an acceleration voltage is induced at the ceramic gap and a circulating beam sees this induced voltage.

FINEMET developed by Hitachi Metal Co. 1td. has been employed as the magnetic core material. FINEMET is a nanocrystalline ribbon made from $\mathrm{Fe}, \mathrm{Si}, \mathrm{B}$, and small amounts of $\mathrm{Cu}$ and $\mathrm{Nb}$. It has a high saturation flux density, high permeability, and good high-frequency characteristics. The core is formed in a bobbin shape with an inner diameter of $225 \mathrm{~mm}$ and an outer diameter of $500 \mathrm{~mm}$ by winding a $13-\mu \mathrm{m}$-thick ribbon around a stainless steel round frame. Six FINEMET cores are stacked in a stainless steel cage. Silicone oil is used for insulation; the oil circulates in a closed loop including a heat exchanger outside the cell. The inner structure of ICs and a photograph of a FINEMET core are shown in Fig. 29, and Fig. 30 shows the assembled acceleration cells which have been installed inside the DA ring.

A practical pulse width available for acceleration is about $500 \mathrm{~ns}$ with an output voltage of $2 \mathrm{kV}$ due to a voltage droop. In order to increase the available pulse length, some type A ICs have been modified to become a 2:1 transformer (type B IC). The primary coil is wound 2 times around the same core, which increases its inductance fourfold reducing the droop. Consequently, $2-\mu$ s acceleration pulses are available from type B ICs [19] at the expense of reducing the output voltage by $1 \mathrm{kV}$.

\section{B. Induction acceleration}

\section{Limitations of acceleration devices}

The revolution period $\left(T_{r}\right)$ and the required accelerating voltage of KEK-DA are calculated from the sinusoidal ramping pattern of the bending magnets described in Sec. IV C. Assuming $\mathrm{Ar}^{8+}, T_{r}$ changes from $13.6 \mu \mathrm{s}$ to $0.753 \mu \mathrm{s}$ as the beam is accelerated. A maximum
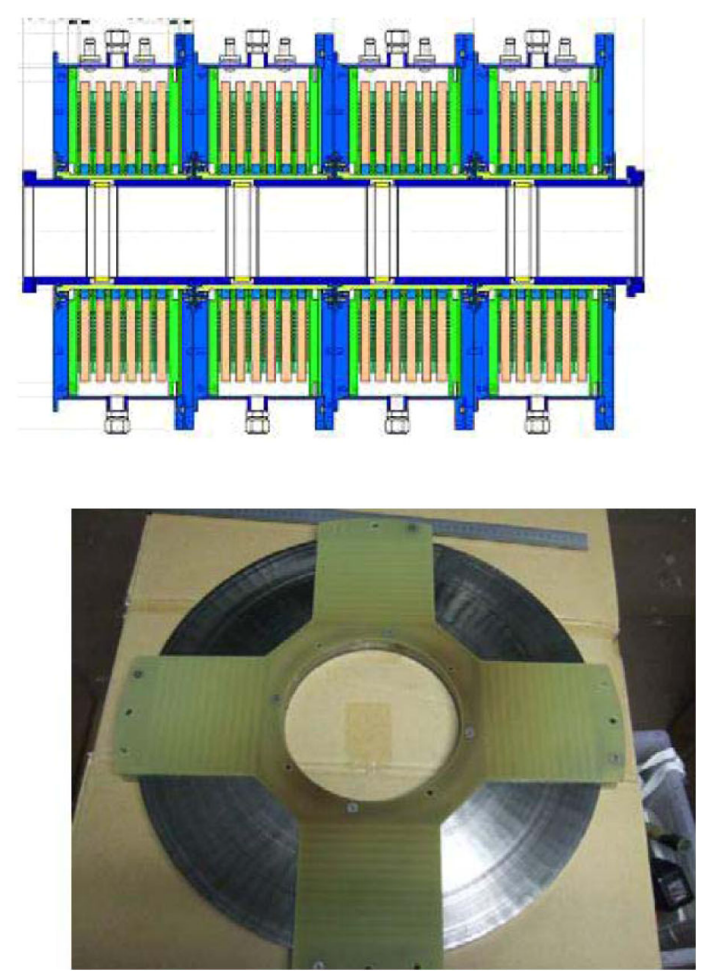

FIG. 29. Inner structure of an IC (top) and a magnetic core bobbin and G-10 spacer with oil flow channels (bottom).

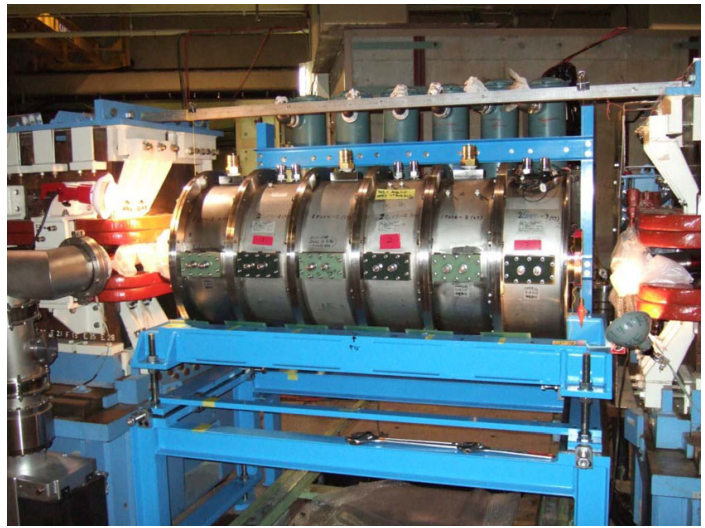

FIG. 30. Acceleration cells installed in the straight section of the KEK-DA.

accelerating voltage of $3 \mathrm{kV}$ is required in the middle of the acceleration cycle. Acceleration devices must meet the strict requirements of high-frequency operation (more than $1 \mathrm{MHz}$ ), a pulse length of order of $4 \mu \mathrm{s}$, and a maximum output voltage of $3 \mathrm{kV}$.

The acceleration devices that had been developed for the induction synchrotron experiment at the KEK-PS and used for the KEK digital accelerator have technical limitations. The output voltage of the DCPS has a maximum value of $2 \mathrm{kV}$ and cannot be varied during the acceleration cycle of $100 \mathrm{~ms}$ due to the circuit parameters. The maximum switching frequency of the SPS is limited to $1 \mathrm{MHz}$ 
because of the cooling capability of the MOSFET element itself.

There is a practical method to meet these requirements even under the existing technical constraints: (i) sequential generation of voltage pulses; (ii) superposition of voltage pulses; and (iii) intermittent operation of multiple induction ICs.

We now describe how the acceleration scheme in the KEK-DA is realized in the above context.

\section{Acceleration scenario}

The acceleration cycle is divided into three stages. Figures 31-33 show a conceptual diagram of the scenario in stages 1 to 3 . In the first stage ( $\left.2 \mu \mathrm{s}<T_{r}<13.6 \mu \mathrm{s}\right)$, a

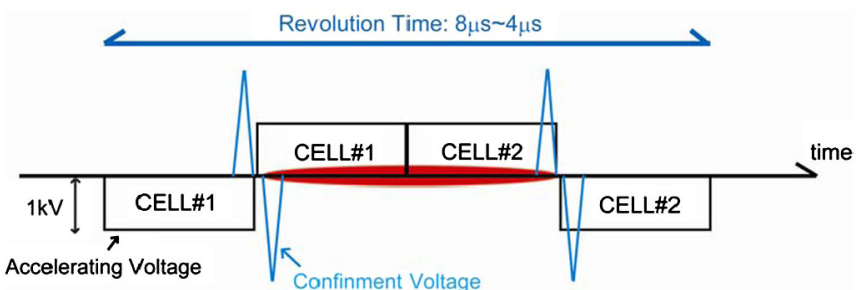

FIG. 31. First stage of acceleration $\left(2 \mu \mathrm{s}<T_{r}<13.6 \mu \mathrm{s}\right)$. To generate a long accelerating voltage, two type B ICs are triggered in series. Pulse shapes shown by the sky blue lines represent the voltage pulses for confinement in the longitudinal direction.

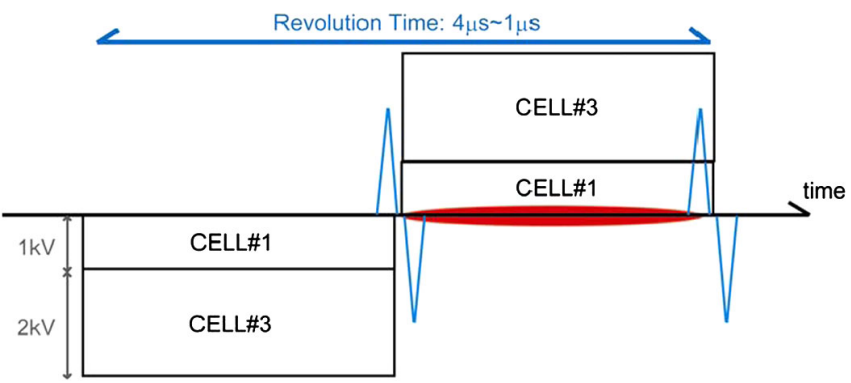

FIG. 32. In the second stage ( $\left.1 \mu \mathrm{s}<T_{r}<2 \mu \mathrm{s}\right)$, a type A and a type B IC are triggered simultaneously.

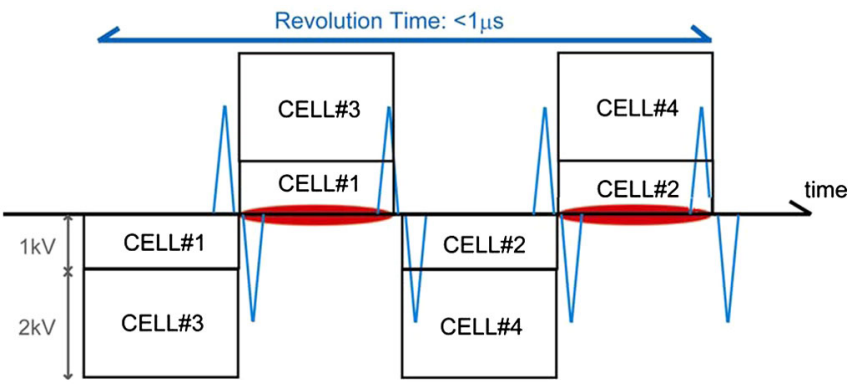

FIG. 33. In the third stage $\left(T_{r}<1 \mu \mathrm{s}\right)$, two pairs of type A and B ICs are operated intermittently. long accelerating voltage pulse is required, as mentioned earlier. Two type B ICs are triggered in series to produce a long voltage pulse. Furthermore, the trigger pulse density of these ICs is controlled so that an integrated accelerating voltage is equal to the required accelerating voltage for a short time. In the second stage $\left(1 \mu \mathrm{s}<T_{r}<2 \mu \mathrm{s}\right)$, a type A and a type B IC are simultaneously triggered to generate a higher effective acceleration voltage. In the third stage $\left(0.753 \mu \mathrm{s}<T_{r}<1 \mu \mathrm{s}\right)$, two pairs of ICs, each consisting of a type A and a type B IC, are intermittently energized.

\section{Beam simulation}

Beam simulation for $\mathrm{Ar}^{8+}$ was carried out using a particle tracking code. The code calculates longitudinal motion in phase space based on assumed DA parameters (ramping magnetic field, acceleration/confinement voltages, momentum aperture, and so on) with realistic perturbations, such as reflection from the other end of the transmission cable. The longitudinal phase space plots at injection and near extraction are shown in Fig. 34. The confinement voltage pulses shown as lines are narrow pulses of $200 \mathrm{~ns}$ width with dual polarities. The width and height of the acceleration voltage pulse is varied as a function of revolution time, as described in the acceleration scenario.
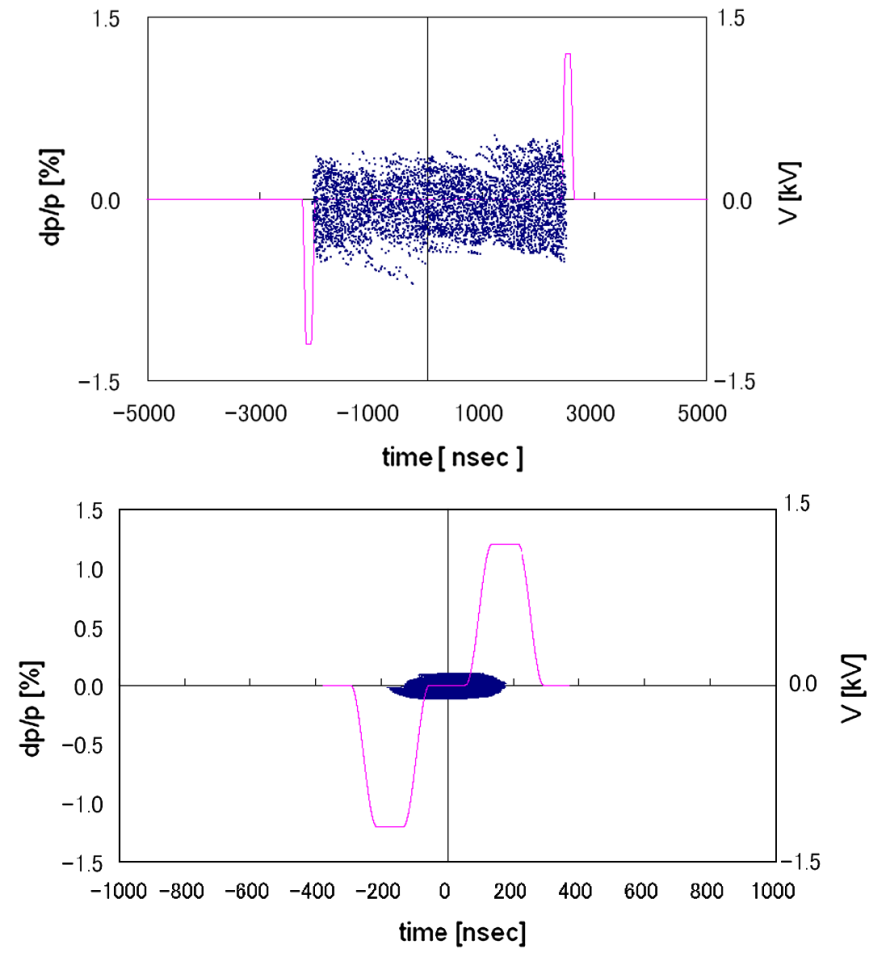

FIG. 34. Phase plot of an $\mathrm{Ar}^{8+}$ beam 100 turns after injection (top) and just before extraction (bottom). Dots show injected ions and the purple lines correspond to barrier voltages for confinement (unit: kV). 


\section{Control system}

\section{Beam bunch monitor}

The gate trigger system of the induction acceleration system triggers the acceleration cells based on the timing signal of the circulating beam bunch, as stated in Sec. I. A nondestructive beam monitor to obtain the timing signal every turn is needed. The magnetic core of our induction cell itself is utilized as a current transformer (CT). A voltage induced by the beam current on the primary loop reflects the beam pulse information. We have examined the properties of this CT. In the one-turn setup, the sensitivity (voltage/current) is about 300, but a large droop was observed. We are developing a droop compensation circuit that amplifies the signal in the low frequency region to monitor the beam bunch profile with high fidelity.

\section{Front-end processing}

From injection to extraction, an ion beam bunch changes its length from $4 \mu$ s to $400 \mathrm{~ns}$. As a result, the pulse height of the beam bunch signal grows tenfold. This feature makes it difficult to set a fixed threshold for the arriving beam pulse height to get beam timing in the noisy environment of the accelerator ring (see Fig. 35). There are other concerns, such as double counting of the beam timing, when the beam pulse has two or more peaks. In order to make the induction acceleration system ready for such dynamic changes in the beam pulse shape, a front-end processing system is essential.

\section{Waveform capturing of beam bunch by analog digital converters $(A D C)$}

Recent developments of ADCs enable us to capture the analog signal in high resolution and at high speed. The extent of the bunch signal height variation is more than 10 times in voltage and the revolution frequency can exceed $1 \mathrm{MHz}$. The required resolution of the ADC is more than 12 bit, taking into account noise effects in the readout circuit. A high-speed 12-bit ADC which works at a sampling rate greater than $100 \mathrm{MS} / \mathrm{s}$ is commercially available. We are preparing a front-end board, on which the

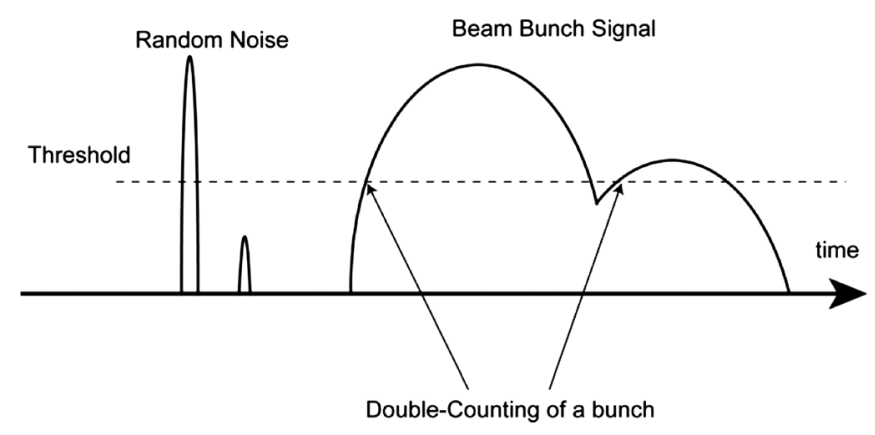

FIG. 35. Difficulties in obtaining beam bunch timing by setting a fixed threshold.
ADC and digital signal processors (DSP) will be mounted, to identify the precise bunch center timing after processing the bunch signal.

\section{Gate trigger system}

In the DA, accelerating voltages must be triggered with a variable delay governed by the bunch signal timing, because the flight time of an ion bunch between the bunch monitor and the ICs, which are separately placed along the accelerator ring, varies with acceleration. Also, the pulse length of accelerating voltages must be gradually changed with acceleration. In addition, in the third stage intermittent operation of the induction acceleration system is required because the revolution frequency exceeds $1 \mathrm{MHz}$ which is a practical limit of the SPS. These requirements are satisfied by controlling the gate trigger signal for the solid-state switching elements. An intelligent gate-control system to fulfill these requirements had been already experimentally demonstrated in the proof-of-principle experiment of induction synchrotron concept [1]. It consists of DSPs and logic circuits, which process beam bunch signals and produce trigger signals for the SPSs. Figure 36 shows an outline of the gate trigger system.

DSPs manufactured by Texas Instruments Co. Ltd. are used as the core device of the intelligent trigger system. The DSP (DSK6416T) is programmed using the C language; it has 32-bit timers with a clock frequency of $128 \mathrm{MHz}$. The program obtains timing information from the ramping pattern of the bending magnets, calculates the necessary delay time, and then generates the trigger pulse.

To change the pulse width of accelerating voltages as the beam accelerates, four DSPs are required for each cell: two

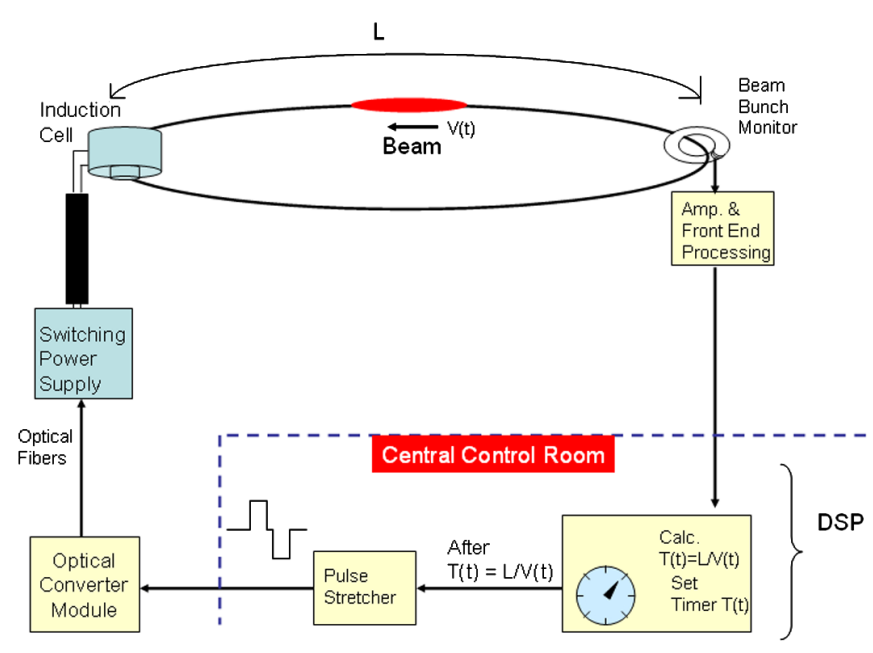

FIG. 36. Outline of the gate trigger system. When a beam with speed $V(t)$ is detected by a beam bunch monitor, the DSP sets its internal timer to make a delayed trigger signal: $T[=L / V(t)]$, where $L$ is the distance between the bunch monitor and the IC, and $t$ denotes the time from the start of acceleration. The pulse stretcher forms the set/reset timing pulse and passes it to the switching power supply. 
for the timing of the start/stop signals and two for the set/ reset pulse voltages. By varying the distance between the start and stop pulses using a pulse stretcher which includes flip-flop circuits, the pulse width can be controlled. Figure 37 shows how four independent DSPs are used to create a pair of set and reset signals.

The revolution frequency is greater than $1 \mathrm{MHz}$ in the third stage, and exceeds the capabilities of the switching

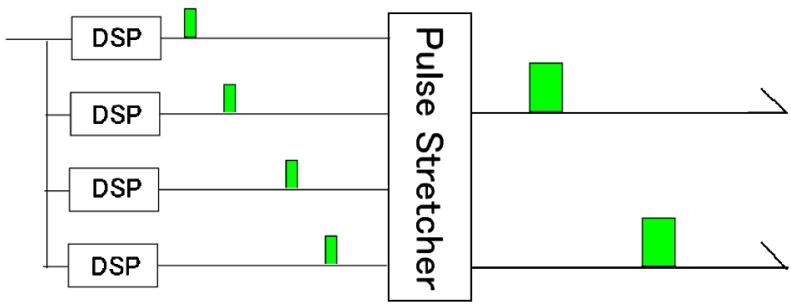

FIG. 37. Pulse stretcher generates set/reset pulse signals with widths that can be controlled by the separations of the input pulses.

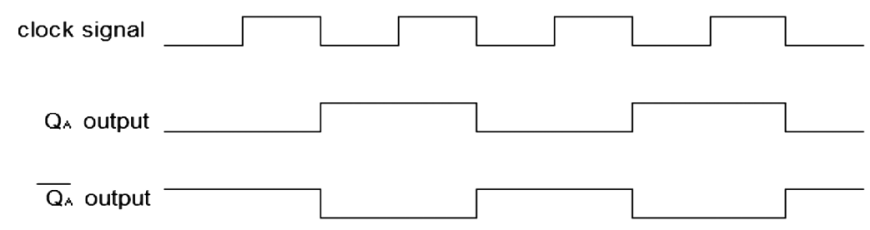

FIG. 38. In the third stage, the signal divider generates gate triggers $Q_{A}$ and $\bar{Q}_{A}$ for two sets of ICs. devices. To avoid a breakdown of the MOSFETs due to excessive heat deposition, one more induction acceleration system joins the operation. A signal divider, which is a combination of frequency divider and multistage logical OR operator (logical disjunction) modules, divides the trigger signal into two parts to energize two ICs. The gate signals pass through the signal divider during the first and second stages. In the third stage, the signal divider blocks every second signal for two sets of cells; in other words, the signal divider triggers two sets of ICs alternately (see Fig. 38).

\section{High-voltage test}

Figure 39 shows high-voltage waveforms controlled by the gate-control system that we have developed. On the left of the plots (stage 1), two ICs are turned on sequentially; on the right, the operation mode is flipped to stage 2 in which ICs are turned on simultaneously. The waveforms in red show the sum of the two output signals that form the effective voltage seen by the beam.

To test the long-term stability of the induction acceleration system, a heat run without beam was performed over one week (from Monday morning to Friday evening, assuming weekday operation). In this heat run six ICs, which is the number expected to be required at the beginning of commissioning, were continuously triggered with a $1 \mathrm{MHz}$ switching frequency. No major problems were encountered in over 100 hours of operation. In Fig. 40 a typical output waveform current observed at the matching resistor (see Fig. 27) is shown.

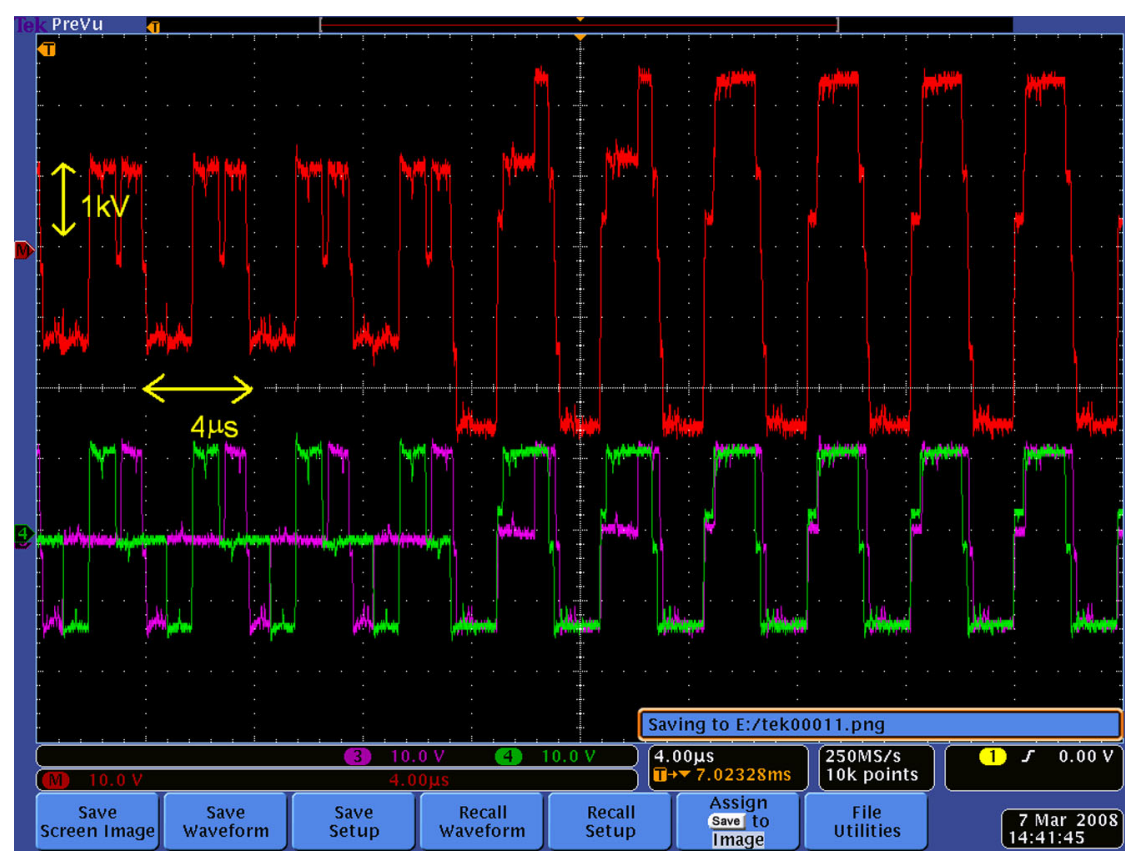

FIG. 39. Output voltage waveform at the transition between stages 1 and 2. (Voltage and time scales are indicated by double-headed arrows.) 


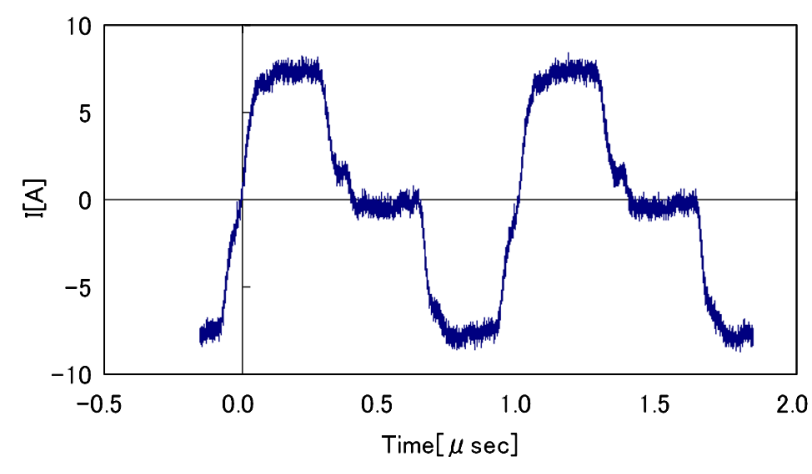

FIG. 40. Output current observed at the matching resistor of the acceleration cell.

\section{BEAM DYNAMICS IN THE DA RING}

\section{A. Space-charge limit}

The number of ions subject to acceleration in the DA is restricted by space-charge effects at injection because of an extremely low $\beta$. Its magnitude can be estimated from the Laslett tune shift,

$$
\Delta \nu \propto \frac{Q^{2} N}{A B_{f} \beta^{2} \gamma^{3}},
$$

where $N$ is the number of charged particles per bunch, $B_{f}$ is the bunching factor, and $Q$ is the charge state of the ion. It is assumed that the beam size is uniquely determined by the acceptance of the aperture. It is believed that $\Delta \nu$ is usually 0.25 in the space-charge limit. Thus, the allowed magnitude of $N$ is known from Eq. (8.1). This number for protons, $N_{p}$, has been found experimentally in the KEK $500 \mathrm{MeV}$ booster. The space-charge limited number of ions per bunch, $N_{i}$, is straightforwardly obtained using $N_{p}$, from

$$
\frac{N_{i}}{N_{p}}=\left(\frac{A}{Q^{2}}\right)\left(\frac{\beta_{i}^{2} \gamma_{i}^{3}}{\beta_{p}^{2} \gamma_{p}^{3}}\right) \frac{\left(B_{f}\right)_{\mathrm{DA}}}{\left(B_{f}\right)_{\mathrm{rf}}} \cong \frac{1}{Q} \frac{\left(B_{f}\right)_{\mathrm{DA}}}{\left(B_{f}\right)_{\mathrm{rf}}},
$$

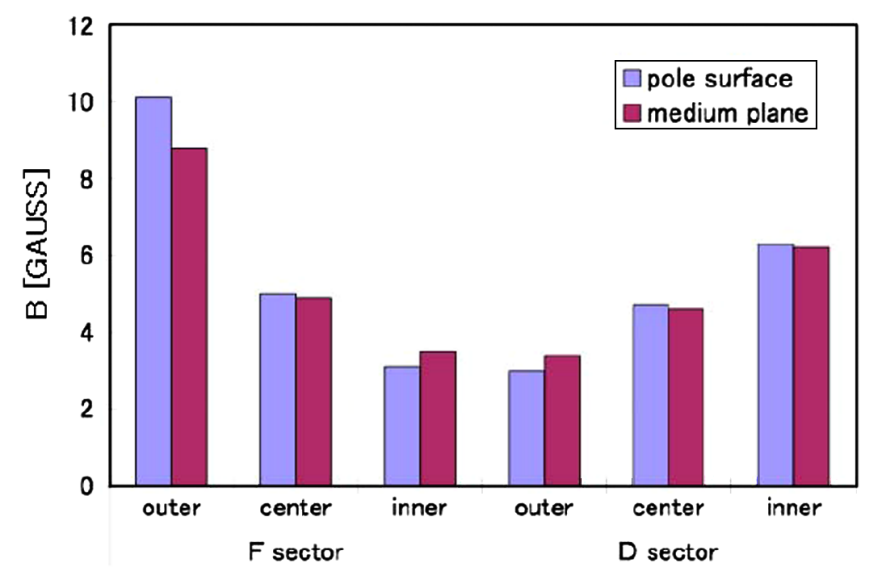

FIG. 41. Measured remanent field of the monitor magnet. where the subscripts $p$ and $i$ denote the parameters for protons and ions, respectively. Here, $\left(B_{f}\right)_{\mathrm{DA}}$ is the bunching factor in the DA, which has been estimated to be $0.4-0.5$. From long experience of the KEK booster operation for protons in the space-charge limited mode, where the injection voltage is $V_{p}=40 \mathrm{MV}$, we know that $N_{p}=$ $3 \times 10^{12}$ and $\left(B_{f}\right)_{\mathrm{rf}}=0.3$. Substituting these values and the injection voltage, $V_{i}=200 \mathrm{kV}$, into Eq. (8.2), we can evaluate the expected space-charge limiting current for typical ions. For ${ }^{40} \mathrm{Ar}^{8+}$, we obtain $N_{i}=4.3 \times 10^{9}$ per bunch, or $4.3 \times 10^{10}$ per second.

\section{B. Remanent fields and closed orbit correction}

Remanent fields in the main magnets have been of concern since we proposed the KEK-DA, because the magnetic flux density is extremely low at injection (about 200-300 Gauss). The remanent fields have been measured at the outer and inner edges of the pole face of all ring magnets, as shown in Fig. 41. The field at the central orbit, which is occupied by a vacuum duct, was estimated by linear interpolation of the measured fields. On the other hand, the remanent fields of the monitor magnet, which is employed for field feedback correction, have been measured at three points (outer edge, middle, and inner edge) along the center axis of the $F$ and $D$ sectors. These results seem to be consistent with each other. According to the measurements, there is a dipole component of $5 \mathrm{G}$ with a maximum $18 \%$ deviation among the magnets. Hence, our estimation of the remanent fields in the ring magnets may have similar deviation.

This field error induces a horizontal closed orbit distortion (COD). Four cases for the remanent field distribution in the ring magnets were prepared, and the COD was calculated for each case. The results show that the possible COD amplitude is about $1 \mathrm{~cm}$, as shown in Fig. 42. COD correction calculations (see Fig. 43) tell us that the magnitude can be reduced to $0.5 \mathrm{~cm}$ by the optimized excitation

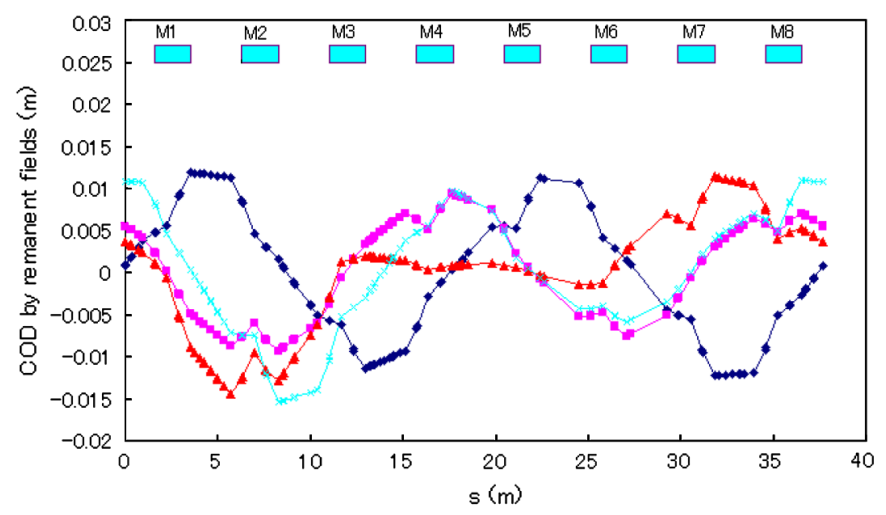

FIG. 42. CODs calculated from the remanent field. Squares at the top of the figure indicate the positions of the eight magnets. Each of the four lines corresponds to one of the cases described in Sec. VIII B. 


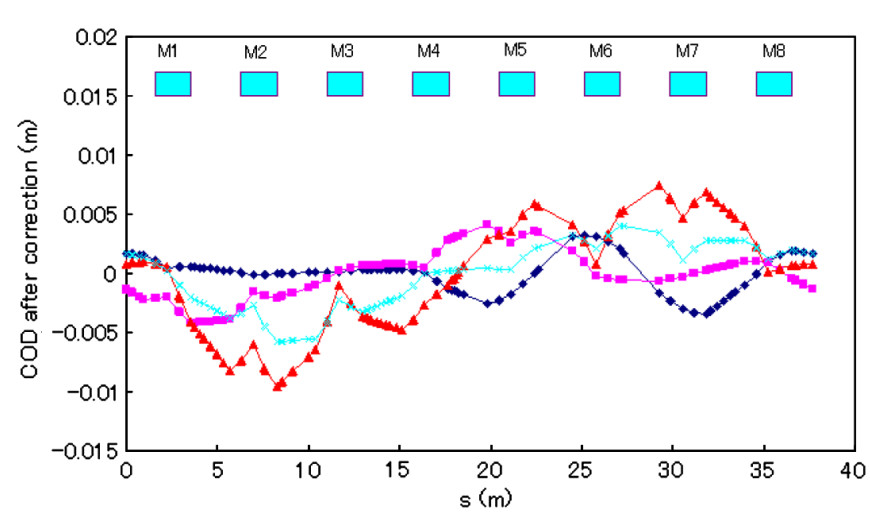

FIG. 43. Corrected CODs.

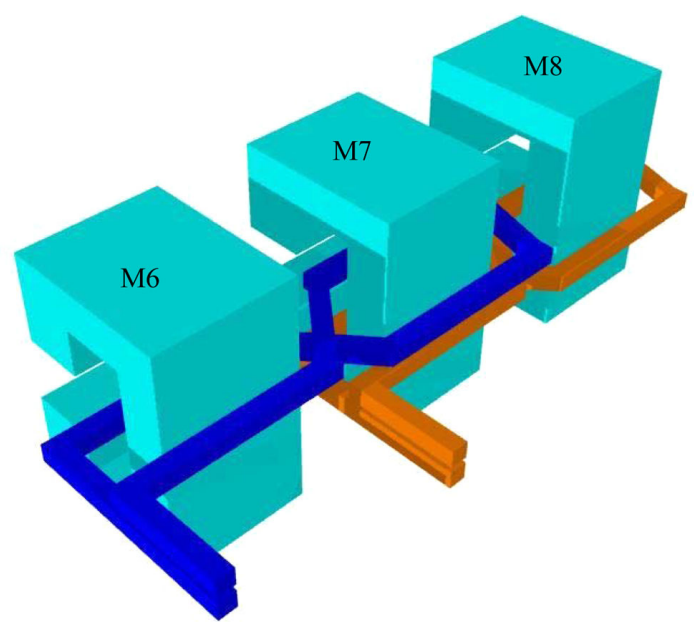

FIG. 44. Two sets of figure-eight back-leg coils for COD correction.

of two sets of the existing figure-eight back-leg coil shown in Fig. 44, which winds around the two poles of adjacent magnets so as to cancel the induced voltage associated with excitation of the main magnet. This size of COD amplitude may be tolerable only for a demonstration of the KEK-DA. However, simulations suggest that using more figure-eight correction systems can reduce the COD further.

\section{Beam loss and vacuum}

It is known that the residual pressure in vacuum chambers of synchrotrons can cause severe loss of particles during acceleration. This loss originates from electron capture or stripping as a result of collisions with residual gas molecules. The two processes are fatal from a beam loss point of view, although other processes, such as Moeller scattering leading to emittance blowup, are also likely to take place. The cross sections of these processes strongly depend on the velocity of the ions and the achieved pressure. Here we describe a simple estimation of beam intensity survival to devise the requirements for the vacuum system design. The $\mathrm{Ar}^{Q+}$ ions are assumed to leave the $200 \mathrm{kV}$ high-voltage terminal with an energy of $200(Q / A) \mathrm{keV} / \mathrm{au}$, where
$A=40$. The probability of capturing one electron is given by the cross section $\sigma_{c}$ and the probability for the loss of an electron by $\sigma_{l}$. The two cross sections are governed by a velocity dependence of different kinds:

$$
\begin{gathered}
\sigma_{C}, \sigma_{l} \sim Q^{p} \beta^{q}, \\
p \sim 2, q \sim\left\{\begin{array}{lll}
-2 & & \text { for } \sigma_{l} \\
-40 & \text { or }-5 & \text { for } \sigma_{C},
\end{array}\right.
\end{gathered}
$$

where $Q$ is the charge state of ion, and $\beta$ is the relativistic factor $v / c$. To simplify calculations, it is generally assumed [19] that

$$
\sigma_{l} \sim Q^{2} \beta^{-2}, \quad \sigma_{C} \sim Q^{2} \beta^{-5} .
$$

It is obviously practical and useful to take actual experimental results, where nitrogen is assumed as a residual gas. The major source of loss is known to be capturing below a few $\mathrm{MeV} / \mathrm{au}$ and stripping beyond that. The survival ratio at time $t$ after injection is given by

$$
S=\frac{n(t)}{n(0)}=\exp \left[-2.12 \times 10^{27} P_{\text {torr }} \int_{0}^{t} \sigma_{\text {tot }} \beta d t\right],
$$

where $\sigma_{\text {tot }}=\sigma_{c}+\sigma_{l}$ and $\beta$ changes with acceleration and is determined from the field ramping pattern. Substituting $\sigma_{l}=\sigma_{l 0}[\beta(0) / \beta(t)]^{2}, \sigma_{c}=\sigma_{c 0}[\beta(0) / \beta(t)]^{5}$, where $\sigma_{l 0}$ and $\sigma_{c 0}$ are derived from the experimental results obtained by Dmitriev et al. [20], gives the survival rate at the end of acceleration. These survival rates are summarized in Table $\mathrm{V}$ as a function of vacuum pressure.

We know that a vacuum pressure of $10^{-7} \mathrm{~Pa}$ is required in order to achieve a survival rate of more than $90 \%$. To realize this requirement, a drastic improvement of the present evacuation system of the KEK-DA is being undertaken. The evacuation capability is enhanced with additional pumps and the evacuated volume along the circulating orbit was reduced by removing the large stainless steel tanks. The septum magnets, which were located in the large size vacuum tank, are now put in air and new ducts inside the magnets have been installed, as described in Sec. V B.

The importance of heavy-ion-induced desorption of gas from vacuum chamber metals has been emphasized in high intensity heavy ion accelerators such as the SIS-18 and SIS-100 of GSI Darmstadt, and the AGS booster and AGS of BNL [21]. It has been reported that the copious desorption associated with the ion bombardment by

TABLE V. Survival rate of the argon beam.

\begin{tabular}{lc}
\hline \hline Vacuum pressure $(\mathrm{Pa})$ & Survival rate \\
\hline $1 \times 10^{-7}$ & 0.95 \\
$5 \times 10^{-7}$ & 0.75 \\
$1 \times 10^{-6}$ & 0.57 \\
$2 \times 10^{-6}$ & 0.32 \\
$4 \times 10^{-6}$ & 0.10 \\
\hline \hline
\end{tabular}


energetic ions on the vacuum chamber surface with a shallow angle leads to dynamic pressure increases. The pressure increase inevitably enhances further beam loss through the mechanism of electron capture and stripping. Effective desorption yields depend on ion species, their energy/nucleon, angle from normal to the target surface. Recent studies [22] have shown that the effective desorption yields clearly depend on electronic energy loss, $\sim\left(d E_{e} / d x\right)^{2}$, in stainless steel. Unfortunately, the effective desorption yield measurements for relatively low-energy Ar ion of current interest are not available. Instead, let us give a rough estimation using that of potassium ions described in Ref. [22], which is placed next to Ar in a periodic table of the elements.

The pressure increase is given by the following equation:

$$
\Delta p=\frac{\eta_{\mathrm{eff}}}{S}\left(\frac{\varepsilon N}{\tau}\right) k_{b} T,
$$

where $\Delta p$ is the pressure increase in Pascal, $\eta_{\text {eff }}$ is the effective desorption yields per ion, $S$ is the pumping speed in $\mathrm{m}^{3} / \mathrm{s}, N$ is the a total number of injected ions per cycle, $\varepsilon$ is the typical loss rate, $\tau$ is the acceleration cycle in sec, $k_{b}$ is the Boltzmann constant $\left(1.38 \times 10^{-23} \mathrm{~J} / \mathrm{K}\right)$, and $T$ is temperature in Kelvin. The kinetic energy of ions at injection is less than $40 \mathrm{keV} / \mathrm{au}$. In this region, $\eta_{\text {eff }}$ is around $10^{3}$ at most. Other parameters are listed in Table VI. Substituting these parameters into the above equation, we have $\Delta p=\varepsilon\left(5.4 \times 10^{-9} \mathrm{~Pa}\right)$. Considering $\varepsilon<1$, we can realize that the pressure increase is still lower than the base pressure of $10^{-7} \mathrm{~Pa}$. In an actual situation, the beam loss might tend to be localized at the limited region. At this moment we do not have enough information for this matter.

\section{POSSIBLE BEAM PARAMETERS AND APPLICATIONS}

As mentioned in the previous section, the ion beam intensity acceptable at the injection stage is limited by

TABLE VI. Parameters of gas desorption.

\begin{tabular}{lc}
\hline \hline$S$ & $6 \times 1000 \mathrm{~L} / \mathrm{s}+3 \times 500 \mathrm{~L} / \mathrm{s}=7.5 \mathrm{~m}^{3} / \mathrm{sec}$ \\
$\tau$ & $10^{-1} \mathrm{sec}$ \\
$N$ & $10^{9}$ \\
$T$ & $293 \mathrm{~K}$ \\
\hline \hline
\end{tabular}

space-charge effects and the survival of the accepted ion intensity depends on the vacuum. Meanwhile, the achieved ion energy is uniquely determined by the maximum magnetic flux density of the guiding magnets and the ion parameter $Q / A$, where $Q$ is the charge state and $A$ is the mass number. The present X-band ECRIS is useful for generating low and medium charge-state ions for gaslike atoms such as $\mathrm{He}, \mathrm{C}, \mathrm{N}, \mathrm{O}, \mathrm{Ne}$, and Ar. Ion parameters that may eventually be achieved are listed in Table VII.

The irradiation of various ions onto metals, magnetic materials, ceramics, semiconducting materials, and polymers has been discussed as a way of developing novel materials, such as nanowires, nanocircuits, and conducting ion tracks in diamondlike carbon [23]. The deep implantation of moderate-energy heavy ions may serve to create a new alloy in bulk because the energy deposition caused by the electroexcitation associated with the passing of highenergy ions through a material greatly modifies its structure [24]. However, the details of the physics dominating the phenomena observed over large scales and long durations are unknown [25]. The KEK-DA will be dedicated to systematic studies to reveal this physics.

In addition, it is planned that the first ion beams from the KEK-DA will be provided to various space chemistry and space radiation technology experiments as pseudo-cosmic rays. The irradiation of various swift ions on dusts of compounds forming space ice, such as ammonia, water, and methanol, is predicted to create various organic substances [26,27]. Results from the infrared spectroscopy of these materials will provide a valuable database for infrared astronomy which is investigating the possible origin of life in space. Damage to solid-state electronics on satellites caused by cosmic ray strikes is also of great concern and the development of radiation-hardened electronics systems is a matter of the utmost urgency. The pinpoint irradiation of various swift ions is expected to take an important role in the analysis of the damage and give useful information for the development of desired electrical systems.

\section{SUMMARY AND PERSPECTIVE}

We have discussed all aspects of the KEK digital accelerator which is capable of accelerating any species of ion in all possible charge states. Most of the components are recycled from the former KEK $500 \mathrm{MeV}$ booster proton

TABLE VII. $\quad$ Ion beam parameters at the first stage of the KEK-DA $\left(B_{r}=0.84(\right.$ Tesla $\left.) \times 3.3(\mathrm{~m})\right)$.

\begin{tabular}{lccccc}
\hline \hline Element & Mass No. $A$ & Charge state $Q$ & $(Q / A)^{2}$ & $E(\mathrm{MeV} / \mathrm{au})$ & Number of ions $(/ \mathrm{s})$ \\
\hline Helium & 4 & 2 & 0.2500 & 102.60 & $7.5 \times 10^{10}$ \\
Carbon & 12 & 4 & 0.1111 & 42.80 & $3.7 \times 10^{10}$ \\
Nitrogen & 14 & 5 & 0.1276 & 49.50 & $3.0 \times 10^{10}$ \\
Oxygen & 16 & 6 & 0.1406 & 54.9 & $2.5 \times 10^{10}$ \\
Neon & 20 & 6 & 0.09 & 34.40 & $2.5 \times 10^{10}$ \\
Argon & 40 & 8 & 0.04 & 15.00 & $1.8 \times 10^{10}$ \\
\hline \hline
\end{tabular}


synchrotron. The main change from the original form is the elimination of the $40 \mathrm{MeV}$ drift tube linac (DTL) and rf cavities. An ECR ion source embedded in the $200 \mathrm{kV}$ highvoltage terminal has been newly developed as an injector, and the rf cavities have been replaced by induction acceleration cells. The low-energy beam transport and newly developed injection/extraction system have been tested and are almost ready. This paper has focused on the induction acceleration system itself and its control because they play a key role for acceleration in the KEK-DA.

We are planning beam commissioning using $\mathrm{He}^{1+}, \mathrm{C}^{4+}$, and $\mathrm{Ar}^{8+}$ in early 2011. If desired, the ions provided can be easily switched to other species such as nitrogen, oxygen, and neon.

\section{ACKNOWLEDGMENTS}

This work was supported by a Grant-In-Aid for Scientific Research (S) (KAKENHI No. 20224005). We thank the following individuals for making valuable contributions to this work: T.S. Dixit (Society for Applied Microwave Electronics Engineering \& Research in India), developing the acceleration scenario in the early stages of R\&D work; K. Zhang (Institute of Fluid Physics), measuring the impedance characteristics of the induction cells; E. Tojyo, T. Wakui, and T. Shinozuka (Tohoku University), providing useful suggestions concerning the ECR ion source; Tadashi Kubo (KEK), providing advice on power receiving and distribution; H. Suzuki (Nippon Advanced Technology), working on preliminary ECRIS development; W. Jiang, providing the idea of a semiconductor switch in the Marx generator for the chopper device; T. Yoshii (Nagaoka Technology University), assisting in testing the injection/extraction system; Y. Sato (KEK), configuring the vacuum system; T. Tachinami for lattice calculations of the LEBT; and T. Tanaka for current measurements at the test bench.

[1] K. Takayama, K. Koseki, K. Torikai, A. Tokuchi, E. Nakamura, Y. Arakida, Y. Shimosaki, M. Wake, T. Kouno, K. Horioka, S. Igarashi, T. Iwashita, A. Kawasaki, J. Kishiro, M. Sakuda, H. Sato, M. Shiho, M. Shirakata, T. Sueno, T. Toyama, M. Watanabe, and I. Yamane, Phys. Rev. Lett. 94, 144801 (2005).

[2] K. Takayama, Y. Arakida, T. Dixit, T. Iwashita, T. Kono, E. Nakamura, K. Otsuka, Y. Shimosaki, K. Torikai, and M. Wake, Phys. Rev. Lett. 98, 054801 (2007).

[3] K. Takayama, in Induction Accelerators edited by $\mathrm{K}$. Takayama and R. Briggs (Springer, Berlin, Heidelberg, 2010), Chaps. 11 and 12.

[4] J. Griffin, C. Ankenbrandt, J. MacLachlan, and A. Moretti, IEEE Trans. Nucl. Sci. 30, 21 (1983).

[5] K. Takayama, Y. Arakida, T. Iwashita, Y. Shimosaki, T. Dixit, and K. Torikai, J. Appl. Phys. 101, 063304 (2007); 103, 099903(E) (2008); Patent No. 3896420, Report No. PCT/JP2006/308502, 2006.
[6] J. Pivarč, J. Electr. Eng. 55, 100 (2004).

[7] S. Gammino and G. Ciavola, Rev. Sci. Instrum. 67, 155 (1996).

[8] R. Becker and W. B. Herrmannsfeldt, Rev. Sci. Instrum. 63, 2756 (1992).

[9] I. G. Brown, The Physics and Technology of Ion Sources (John Wiley \& Sons, Hoboken, 2004), 2nd ed.), ISBN 3-527-40410-4.

[10] T. Adachi, T. Arai, K. W. Leo, K. Takayama, and A. Tokuchi, "A Solid-state Marx Generator driven Einzellens Chopper" (unpublished).

[11] Nisshin Ion Equipment Co., Ltd., EXCEED2000A HVT with acceleration column.

[12] Femtet $($ C Murata Software Co., Ltd.

[13] H. Sasaki, K. Takikawa, and M. Kumada, KEK Report No. KEK-73-2.

[14] Y. Arakida, T. Inagaki, T. Kawakubo, Y. Kimura, S. Mitsunobu, K. Satoh, K. Takata, S. Hazawa, and S. Tokumoto, in Proceedings of PAC1977 [IEEE Trans. Nucl. Sci. 24, No. 3 (1977)], p. 1464.

[15] T. Adachi, T. Kawakubo, and T. Yoshii, in Proceedings of IPAC'10 (2010), MOPEC050, p. 570-572.

[16] M. Wake, Y. Arakida, K. Koseki, Y. Shimosaki, K. Takayama, K. Torikai, W. Jiang, K. Nakahiro, A. Tokuchi, and A. Sugiyama, in Proceedings of the 2007 Particle Accelerator Conference, Albuquerque, New Mexico (IEEE, New York, 2007), MOPAN042, p. 251253.

[17] K. Koseki, M. Wake, and K. Takayama, Nucl. Instrum. Methods Phys. Res., Sect. A 554, 64 (2005).

[18] T. S. Dixit, T. Iwashita, and K. Takayama, Nucl. Instrum. Methods Phys. Res., Sect. A 602, 326 (2009).

[19] Dr. Jean-Michel Lagniel (private communications).

[20] I. S. Dmitriev, Ya. A. Teplova, Yu. A. Belkova, N. V. Novikov, and Yu. A. Fainberg, At. Data Nucl. Data Tables 96, 85 (2010).

[21] S. Y. Zhang and L. A. Ahrens, in Proceedings of the 18th Particle Accelerator Conference, New York, 1999 (IEEE, New York, 1999), p. 3294-3296; C. Omet, P. Spillar, and J. Stadlmann, in Proceedings of the 10th European Particle Accelerator Conference, Edinburgh, Scotland, 2006 (EPS-AG, Edinburgh, Scotland, 2006), p. 211-213; W. Fisher et al., in Proceedings of the 11th European Particle Accelerator Conference, Genoa, 2008 (EPS-AG, Genoa, Italy, 2008), p. 313-315.

[22] A. W. Molvik et al., Phys. Rev. Lett. 98, 064801 (2007).

[23] A. Weidinger, Europhysics News 35, 152 (2004).

[24] U. Glasmacher, M. Lang, H. Keppler, F. Langeonhorst, R. Neumann, D. Schardt, C. Trautmann, and G. Wagner, Phys. Rev. Lett. 96, 195701 (2006).

[25] A. Iwase et al., Nucl. Instrum. Methods Phys. Res., Sect. B 195, 309 (2002).

[26] G. M. Muñoz Caro, U. J. Meierhenrich, W. A. Schutte1, B. Barbier, A. Arcones Segovia, H. Rosenbauer, W. H.-P. Thiemann, A. Brack, and J.M. Greenberg, Nature (London) 416, 403 (2002).

[27] Muriel Gargaud, Jose Cernicharo, Michel Viso, Henderson James Cleaves II, Daniele Pinti, Ricardo Amils, and Kensei Kobayashi, Encyclopedia of Astrobiology (Springer, Berlin, Heidelberg, 2011). 